Supplement of

\title{
Regional new particle formation as modulators of cloud condensation nuclei and cloud droplet number in the eastern Mediterranean
}

Panayiotis Kalkavouras et al.

Correspondence to: Athanasios Nenes (athanasios.nenes@epfl.ch) and Aikaterini Bougiatioti (abougiat@noa.gr)

The copyright of individual parts of the supplement might differ from the CC BY 4.0 License. 


\section{SM 3.2 Impact of NPF on CCN concentrations and total aerosol number}

Seasonally, 24 wintertime NPF events were recorded at Finokalia between 4:50 and 14:45 LT, with subsequent growth of the newly formed particles over the rest of the day. The analysis of the air-mass back-trajectories shows that the air originated from the North for 12 events with average speeds $7.0 \pm 0.1 \mathrm{~m} \mathrm{~s}^{-1}$, characterized by clean air with low pollutant concentrations. Prior to initiation of the NPF, the diurnal mean value of calculated $\mathrm{N}_{\text {total }}$ were $2,183 \pm 134 \mathrm{~cm}^{-3}$, and $3,902 \pm 993 \mathrm{~cm}^{-3}$ when NPF ends, resulting in an increase of almost $79 \%$. 9 events took place when air-masses originated from the West/Southwest sector, coming from North Africa, and afterwards spending time over the island of Crete, with moderate speeds $\left(5.2 \pm 1.1 \mathrm{~m} \mathrm{~s}^{-1}\right)$. The events were characterized by a strong increase in the diurnal mean value of $\mathrm{N}_{\text {total }}(156 \%)$, corresponding to $1,578 \pm 387 \mathrm{~cm}^{-3}$ before, and $4,037 \pm 1,120 \mathrm{~cm}^{-3}$ after the NPF. Only 3 events were recorded when eastern winds prevailed over the Finokalia station, with speeds above even $11 \mathrm{~m} \mathrm{~s}^{-1}$ (average $9.6 \pm 2.9 \mathrm{~m} \mathrm{~s}^{-1}$ ), and a diurnal mean increase of $\mathrm{N}_{\text {total }}$ by $286 \%$. To identify the contribution of atmospheric NPF episodes to the production of new CCN during winter, we focus on the $t_{\text {dec }}$ (varying from 0.42 to $8.5 \mathrm{~h}$, with an average value of $2.9 \pm 2.2 \mathrm{~h}$ ), where the $R_{\mathrm{S}}$ display a sharp increase few hours after the $t_{\text {start. }}$. The calculated $R_{\mathrm{S}}$ was found to range between 0.64 and 2.87 (mean value $1.17 \pm 0.40$ ) before $t_{d e c}$, and 0.63 to 5.61 (mean value $1.71 \pm 0.92$ ) before and after the $t_{d e c}$, respectively, and the CCN population exhibits an increase of $30,43,43,44,46$, and $54 \%$, respectively for each $s$. Given that smaller particles activate earlier to CCN size range particles, the $t_{d e c}$ is similar at each supersaturation level, which indicates that the size of the particles ( $d_{c}$ varying from 29 to $216 \mathrm{~nm}$ ) is negligible in order to note a different $t_{\text {dec }}$.

In spring $\mathrm{N}_{\text {total }}$ was about $8 \%$ higher than in winter (average $\mathrm{N}_{\text {total }}=3,215 \pm 807 \mathrm{~cm}^{-3}$ ), with distinct air-mass origin during the NPF episodes (Table S2). 35 events were recorded, with the $t_{\text {start }}$ ranging between 7:40 and 13:15 LT, ending up with high wind speeds (average $7.2 \pm 0.4 \mathrm{~m} \mathrm{~s}^{-1}$ ) from the North sector $\left(315^{\circ}\right.$ to $\left.45^{\circ}\right)$, while 17 were detected between 7:15 and 13:00 LT, when air-masses arriving at Finokalia with an average wind speed $3.0 \pm 0.3 \mathrm{~m} \mathrm{~s}^{-1}$ from the West/Southwest sector $\left(270^{\circ}\right.$ to $\left.200^{\circ}\right)$, firstly spending time over Crete and thus being influenced by the island. The days where the events trigger the CCN production are characterized by moderate humidity (RH 60\%), and spring temperature exhibited values larger than $17.5{ }^{\circ} \mathrm{C}$ between $10: 00$ and 17:00 LT. Before the $t_{\text {start }}$, the diurnal average estimated value of $\mathrm{N}_{\text {total }}$ was $2,701 \pm 102 \mathrm{~cm}^{-3}$, and $2,362 \pm 142 \mathrm{~cm}^{-3}$ under northern and south/southwestern winds, respectively, while after the subsequent condensational growth of the freshly-formed particles the $\mathrm{N}_{\text {total }}$ was $4,103 \pm 500 \mathrm{~cm}^{-3}$, and $3,631 \pm 580 \mathrm{~cm}^{-3}$ for the aforementioned air-mass source regions. Therefore, it seems that higher aerosol number concentrations of new particles were observed, when air-masses came from the marine 
boundary layer (northern directions), indicating that the sources of nucleating and condensing species are stronger over the marine environment than over the land (Tunved et al., 2006). The time evolution of the $R_{\mathrm{s}}$ in spring displays a diurnal variability. The contribution of new aerosols on CCN generally occurs between 10 minutes and 11 hours (average $2.6 \pm 1.8 \mathrm{~h}$ ) after the beginning of the particle formation events $\left(t_{\text {start }}\right)$, promoting the growth of the new particles. The $R_{\mathrm{S}}$ ranges from 0.37 to 2.21 for all supersaturations during the period prior to the beginning of the production CCN (see Table S2), and they are constantly similar for each supersaturation (mean value $1.09 \pm 0.28$ ) before the $t_{d e c}$, which suggest that at a given supersaturation level the predicted CCN values stay quite constant until the "wave" of fresh particle reaches to sufficient size to affect CCN. Via the values of the $R_{\mathrm{s}}$ we observed that NPF contributes significantly to CCN concentrations by 33, 41, 41, 43, 43, and 45\%, for each supersaturation. Since the influence is almost similar for $s 0.10,0.38,0.52,0.66,0.73$, and $1.00 \%$ reveals that, smaller (average $d_{c}=35 \mathrm{~nm}$ at $s=1.00 \%$ ) and large (average $d_{c}=160 \mathrm{~nm}$ at $s=0.38 \%$ ) particles "feel" all the same the NPF, supporting that the enhancement of the CCN concentrations was not only atmospheric nucleation, but in addition the growth of sub-CCNsized primary particles throughout their transportation.

Moving to the summer episodes, the average number concentrations of aerosol particles was calculated to be $4,254 \pm 372 \mathrm{~cm}^{-3}$, which is the highest of all seasons. 50 daytime NPF events were recorded, occurring between 6:45 and 11:05 LT. The air-mass back-trajectories in conjunction with the wind direction data indicates that out of the 50 events, the grand majority (43 events) were associated with strong northerlies over the Aegean Sea, with speeds above $8 \mathrm{~m} \mathrm{~s}^{-1}$ (Etesian flow), while the rest 7 episodes took place when air-masses originated from the wider West/Southwest sector. Furthermore, apart from the strong winds, the summer temperatures vary between 18 and $31^{\circ} \mathrm{C}$, and this season is considered as humid, with RH values reaching up to $75 \%$ at the Finokalia station. Regarding now the Etesian flow, NPF possibly did not initiate in the vicinity of Finokalia, but probably had been taken place a few hours before air-masses arrive at the station. The freshly-formed particles were recorded at the lowest detectable sizes $(\sim 10 \mathrm{~nm})$, with subsequent growth over the rest of the day, and these events are typical examples of so-called regional NPF events, in which the growth homogeneously takes place over distances of hundreds of kilometers (Kulmala et al., 2012; Kalkavouras et al., 2017). The diurnal mean value of $\mathrm{N}_{\text {total }}$ during summer was determined to be $3,681 \pm 174 \mathrm{~cm}^{-3}$ prior to the growth of the particles in the nucleation-mode, and 4,392 \pm 322 $\mathrm{cm}^{-3}$ after the starting of NPF, exhibiting a 20\% increase, which is related to regional sources of pollutants via strong transport from the main European continent and Istanbul (Gerasopoulos et al., 2005; Kalkavouras et al., 2017). The diurnal mean value of the ratio $R_{\mathrm{s}}$ calculated throughout summer was $1.02 \pm 0.24$ before the starting of CCN influence, which is 
the lowest seasonal value. The increase in the aerosol number concentration, $\mathrm{N}_{\text {total }}$, caused by nucleation, was followed by an increase in the $R_{\mathrm{s}}$ with a time lag between 40 minutes and 9.5 hours (mean duration between $t_{\text {start }}$ and $t_{\text {dec }}$ was calculated to be $2.75 \mathrm{~h}$ ), due to the gradual growth of freshly-formed particles up to $25 \mathrm{~nm}$ during the rest of the day. The time evolution of the $R_{\mathrm{s}}$ resembled that of $\mathrm{N}_{\text {total }}$, and the contribution to the CCN concentrations were 38,38 , $39,41,43$, and $45 \%$ (mean $41 \%$ ) at each supersaturation, respectively. It can be seen that, new aerosol particles enhance the CCN concentrations to a slight extent at supersaturations above $0.52 \%$, and therefore the similar values of the percentages likely reflect the atmospheric aging (through condensation/coagulation and cloud processing) of small ( $d_{c}$ varied between 28 and $40 \mathrm{~nm}$ ), less CCN-active aerosol directly formed from NPF events.

In autumn the relative reduction of $\mathrm{N}_{\text {total }}$ compared to summer was $42 \%$ (mean value: $\mathrm{N}_{\text {total }}=$ 2,997 $\pm 662 \mathrm{~cm}^{-3}$ ), and the back-trajectory analysis of the air-masses sampled at Finokalia indicates different source regions from the other seasons. 11 NPF events were recorded between 8:25 and 12:25 LT, arriving with low wind speed (mean $4.4 \pm 0.2 \mathrm{~m} \mathrm{~s}^{-1}$ ) from the west and south/southwest $\left(270^{\circ}\right.$ to $\left.200^{\circ}\right), 24 \mathrm{NPF}$ episodes were observed between 5:30 and 13:00 LT, when air-masses arriving at Finokalia from the north $\left(310^{\circ}\right.$ to $\left.50^{\circ}\right)$ with an average wind speed $7 \pm 0.5 \mathrm{~m} \mathrm{~s}^{-1}$, and a NPF event was detected when the air-mass coming from east. Prior to the $t_{\text {start }}$, the diurnal mean calculated value of $\mathrm{N}_{\text {total }}$ was $2,752 \pm 96 \mathrm{~cm}^{-3}$, and $1,544 \pm 104 \mathrm{~cm}^{-3}$ under northern and south/southwestern air-masses, respectively, while after the subsequent condensational growth of the new particles the $N_{\text {total }}$ was $3,829 \pm 585 \mathrm{~cm}^{-3}$, and $2,691 \pm 603 \mathrm{~cm}^{-3}$ for the aforementioned air-masses origin. Thus, it appears that $\mathrm{N}_{\text {total }}$ has a significantly similar behavior for air-masses coming from the Balkans, and for air-masses originating from Northern Africa. Concerning finally the fraction of the $R_{\mathrm{S}}$ in autumn, the mean value for all episodes was determined as being $1.10 \pm 0.23$ concerning all supersaturations before the $t_{\text {dec }}$, while the average value of the $R_{\mathrm{s}}$ was calculated $1.73 \pm 0.98$, on average 3.3 hours after the $t_{\text {start }}$ ( $t_{\text {dec }}$ between 8:45 and 17:45 LT). Using the values of the $R_{\mathrm{s}}$, we determined that NPF increases significantly to CCN concentrations by $29,47,51,55,58$, and $77 \%$, for each supersaturation. In autumn the largest delay in observing CCN impacts - $3.5 \mathrm{~h}$ after the start of the event - promote the gradually condensational growth of the new particles, which leads to a significantly increase on estimated CCN levels compared to the other seasons, mainly for higher levels of $s$ (0.52 to $1.00 \%)$.

\section{SM 3.3 Impact of NPF on droplet number and cloud formation}

Considering as a fact that the above NPF episode considerably boosts the CCN concentrations could be misleading as to the impact on CDNCs, since the effect of NPF on CCN and therefore on $\mathrm{N}_{\mathrm{d}}$ should not be treated separately. The actual increase caused by the impact of NPF on $\mathrm{N}_{\mathrm{d}}$ would be the same as the impact on CCN but at a supersaturation level equal to 
$s_{\max }$, suggesting that using solely the CCN-based analysis is useless if someone really wants to quantify the influence of NPF on $\mathrm{N}_{\mathrm{d}}$. Throughout the NPF, the $s_{\max }$ which formed in a cloud actually slowly increases after the initiation of NPF and decreases gradually when the "wave" of new particles influences the $\mathrm{N}_{\mathrm{d}}$. Moreover, the $\mathrm{N}_{\mathrm{d}}$ does not increase but only until late in the afternoon, owing to the enhanced competition for water vapor by the ever increasing CCN. The mean value of the $s_{\max }$ was determined as being $0.11 \pm 0.03 \%$, and $0.15 \pm 0.05 \%$ for updraft velocities of $0.3 \mathrm{~m} \mathrm{~s}^{-1}$, and $0.6 \mathrm{~m} \mathrm{~s}^{-1}$, respectively which could be proposed as indicative values when someone uses parameterizations to calculate the $\mathrm{N}_{\mathrm{d}}$. Nevertheless, by selecting a pre-determined supersaturation or a critical diameter to calculate CCN concentrations and $\mathrm{N}_{\mathrm{d}}$, can lead to an ambiguous conclusion regarding the influence of NPF on ambient clouds, and would be preferable to avoid the CCN-based approach and instead refer to the new paradigm of analysis that uses only parameterizations and $\mathrm{N}_{\mathrm{d}}$.

During winter, all NPF events $(n=24)$ over Finokalia trigger the production of CCN and therefore the formation of $\mathrm{N}_{\mathrm{d}}$ between 8:05 and 17:40 LT, on average 2.6 hours after the $t_{\text {dec }}$ ( $t_{N d}$ was recorded from $30 \mathrm{~min}$ to 6 hours, after the $t_{\text {dec }}$ ). For both $\sigma_{w}$ a slight decrease regarding the $s_{\max }$ was calculated compared to the period between $t_{\text {start }}$ and $t_{d e c}$, while the respective perturbation regarding the $\mathrm{N}_{\mathrm{d}}$ from the NPF episodes exhibits on average a limited increase (see Table S3). The supersaturations developed in NPF-influenced clouds ( $0.13 \%$ for $0.3 \mathrm{~m} \mathrm{~s}^{-1}$ and $0.18 \%$ for $0.6 \mathrm{~m} \mathrm{~s}^{-1}$ ) in winter, moves the size of particles affected by cloud processing to larger particles, since the critical diameters during the NPF events varied from 86 to $190 \mathrm{~nm}$ (mean $d_{c} 150 \mathrm{~nm}$ for supersaturation $0.10 \%$, and $95 \mathrm{~nm}$ for $0.20 \%$ ). Moreover, the percentage change of the variance in $\mathrm{N}_{\mathrm{d}}$ for both updraft velocities $\sigma_{\mathrm{w}}$, exhibits the highest seasonal variance regarding the $\mathrm{N}_{\mathrm{d}}$, which is in conjunction with the lowest variability in $\mathrm{N}_{\text {total. }}$. From the relative contribution of the total aerosol number and chemical composition to $\mathrm{N}_{\mathrm{d}}$, it can be seen that the larger contribution is owed to the variance of the total aerosol number to the potential CDNC, while the influence of the chemical composition being limited for both cloud updraft velocities (Table S5).

Throughout spring, aerosol levels were much higher compared to winter and $\mathrm{N}_{\mathrm{d}}$ exhibited also an increasing trend after the NPF episodes. All events ( $\mathrm{n}=52)$ occurred during daytime, and the enhancement of the $\mathrm{N}_{\mathrm{d}}$ observed from 9:25 to 21:45 LT, on average 3 hours after the $t_{\text {dec }}$ ( $t_{N d}$ was varied between 40 min and 8.5 hours, after the $t_{d e c}$ ). Overall, the contribution of the NPF episodes concerning the cloud droplet number concentrations increased as expected for $\sigma_{w}=0.3$ and $0.6 \mathrm{~m} \mathrm{~s}^{-1}$, respectively compared to pre- $t_{N d}$ values (Table S3). This augmentation is related with the decrease in $s_{\max }$, for the aforementioned cloud updraft velocities, which indicates that the increase of competition of CCN for water vapor with $d_{c}$ varying from 29 to $200 \mathrm{~nm}$, can reduce cloud supersaturation. The variance in the $\mathrm{N}_{\mathrm{d}}$ caused by the NPF 
corresponds to a variability in $\mathrm{N}_{\text {total }}$ of more than 1,600 particles $\left(\mathrm{cm}^{-3}\right)$. On average, in spring almost the same values are calculated as during winter regarding the percentage contribution of the total aerosol number and hygroscopicity to the daily droplet number variance calculated for both $\sigma_{w}$ (Table S5). This further supports the above finding that the droplet number variability mostly reflects the intensity of the total aerosol number over Finokalia during NPF events.

The relative contribution of NPF into CDNC during summer was observed on average 3.1 hours after the initiation of potential CCN production. Particularly, $t_{N d}$ occurred mainly after 12:00 LT (47 days), apart from 3 days when $t_{N d}$ was specified between 10:45 to 11:50 LT. Concerning now the $s_{\max }$, it decreases for both cloud updraft velocities, compared to the period between $t_{\text {start }}$ and $t_{\text {dec }}$, once again, and it should be mentioned that, the doubling in the $\sigma_{w}$ causes $s_{\max }$ to increase by $43 \%$. The decrease caused by the NPF was on average all the same, when $\sigma_{w}$ is equal to 0.3 and $0.6 \mathrm{~m} \mathrm{~s}^{-1}$, which indicates that the fluctuation of $s_{\max }$ is independent from the larger cloud updraft velocity, allowing the newly formed particles, which in the meanwhile grow to larger particles (155 nm; accumulation-mode) to activate into cloud droplets. As expected, the reduction of $s_{\max }$ goes with a respective increase of $\mathrm{N}_{\mathrm{d}}$ during the NPF days. For all events in summer, the NPF is followed by a limited augmentation regarding the $\mathrm{N}_{\mathrm{d}}$ (Table S3), regardless of the highest seasonal aerosol concentrations (mean $4,254 \pm 372 \mathrm{~cm}^{-3}$ ), and by extension the highest estimated CCN concentrations for each supersaturation observed during this season, confirming once again that larger and more aged aerosol particles are mostly affected and may activate into cloud droplets. The variance of CDNC due to NPF was determined to be merely 36 and $101 \mathrm{~cm}^{-3}$ for each $\sigma_{w}$, whereas the highest contribution to the variance of $\mathrm{N}_{\mathrm{d}}$ is attributed once more to aerosol number and to a limited extent to the chemical composition. By doubling the cloud updraft velocity, the chemical composition during summer exhibits the highest contribution to CDNC compared to the other seasons, indicating the enhancement of vertical mixing which preceded the arrival of the air plume at Finokalia.

In autumn the NPF events $(n=36)$ influenced the CDNC between 10:15 and 21:10 LT, on average 2.8 hours after the $t_{\text {dec }}$. When $\sigma_{w}$ is equal to $0.3 \mathrm{~m} \mathrm{~s}^{-1}$ the NPF episodes are associated with $s_{\max }$ decreases compared to the period between $t_{\text {start }}$ and $t_{\text {dec }}$ (Table S3), and in consequence, CDNC throughout the NPF events increased, compared to the pre- $t_{N d}$ values. The larger $\sigma_{w}$ lead to higher values of $s_{\max }$ showing an increase of $50 \%$, which allow smaller particles to activate into droplets. $\mathrm{N}_{\mathrm{d}}$ displays a substantial augmentation for $\sigma_{w}=0.6 \mathrm{~m} \mathrm{~s}^{-1}$ (on average $52 \%$ ), indicating the impact of mean vertical velocity on the CDNC. The mean $\mathrm{N}_{\mathrm{d}}$ concentrations prior and after the $t_{N d}$, exhibited relative increase of $15 \%$. The respective 
variance of $\mathrm{N}_{\mathrm{d}}$ when the NPF influence CDNC was 91 and $205 \mathrm{~cm}^{-3}$ for both $\sigma_{w}$, respectively while the predicted $\mathrm{N}_{\mathrm{d}}$ variability is almost entirely controlled by the aerosol number fluctuation, and to a limited extent by the chemical composition for both cloud updraft velocities.

\section{References}

Gerasopoulos, E., Kouvarakis, G., Vrekoussis, M., Kanakidou, M., and Mihalopoulos, N.: Ozone variability in the marine boundary layer of the eastern Mediterranean based on 7-year observations, J. Geophys. Res.-Atmos., 110, D15309, doi:10.1029/2005JD005991, 2005.

Kalkavouras, P., Bossioli, E., Bezantakos, S., Bougiatioti, A., Kalivitis, N., Stavroulas, I., Kouvarakis, G., Protonotariou, A.P., Dandou, A., Biskos, G., Mihalopoulos, N., Nenes, A., and Tombrou, M.: New particle formation in the southern Aegean Sea during the Etesians: importance for CCN production and cloud droplet number, Atmos. Chem. Phys.,17, 175192,doi:10.5194/acp-17-175-2017, 2017.

Kulmala, M., Petäjä, T., Nieminen, T., Sipilä, M., Manninen, H.E., Lehtipalo, K., Dal Maso, M., Aalto, P., Junninen, H., Paasonen, P., Riipinen, I., Lehtinen, K.E.J., Laaksonen, A., and Kerminen, V.-M.: Measurement of the nucleation of atmospheric aerosol particles, Nat. Protocol., 7, 1651-1667, doi:10.1038/nprot.2012.091, 2012.

Tunved, P., Hansson, H.-C., Kerminen, V.-M., Ström, J., Dal Maso, M., Lihavainen, H., Viisanen, Y., Aalto, P.P., Komppula, M., and Kulmala, M.: High Natural Aerosol Loading Over Boreal Forests, Science, 312, 261-263, doi:10.1126/science.1123052, 2006. 


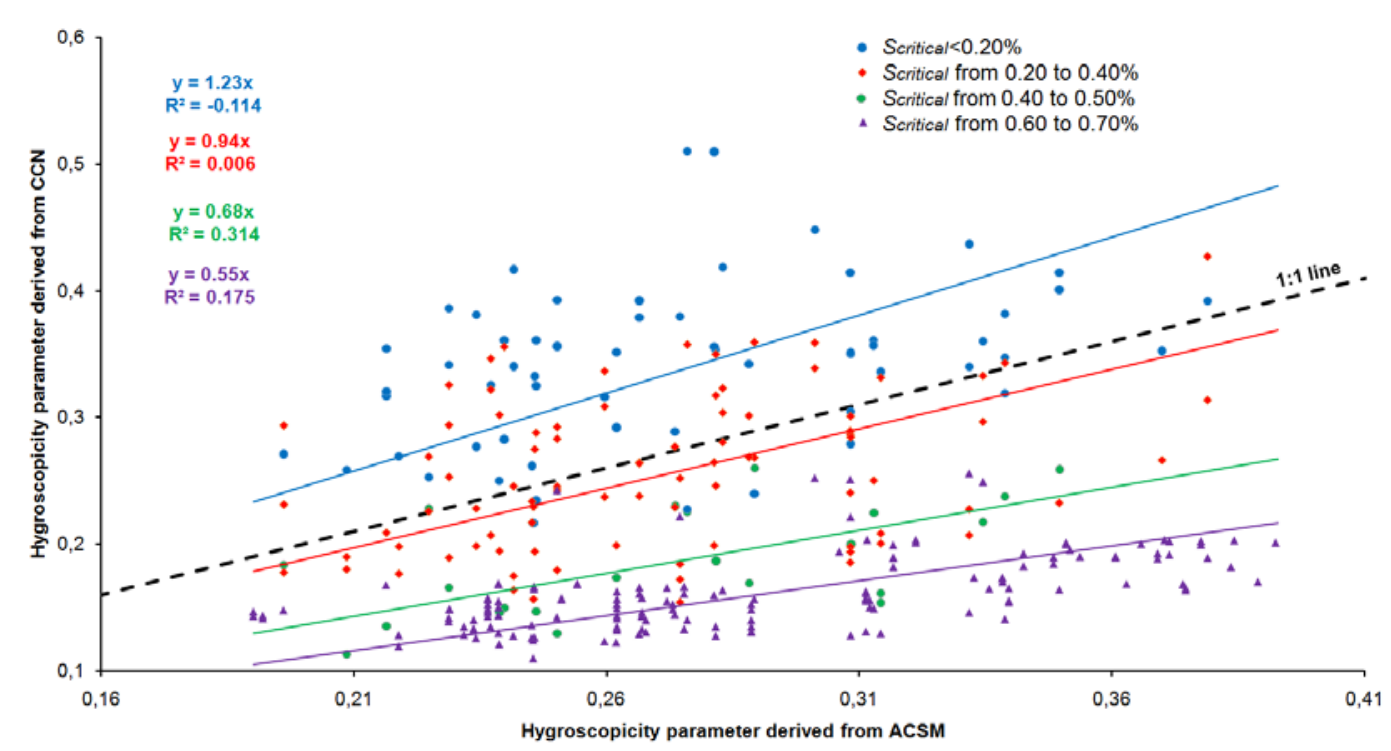

Figure S1. Predicted against measured hygroscopicity parameter $(\kappa)$ at critical supersaturation under $0.20 \%$ (blue dot), from 0.20 to $0.40 \%$ (red rhombus), from 0.40 to $0.50 \%$ (green dot), and from 0.60 to $0.70 \%$ (purple triangle), respectively on 29 , 30 August and 9, 22 September 2012.

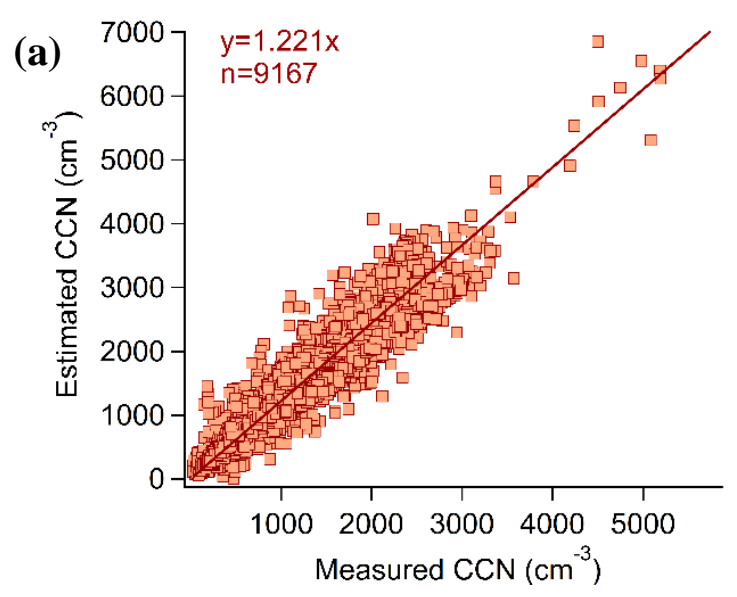

(b)

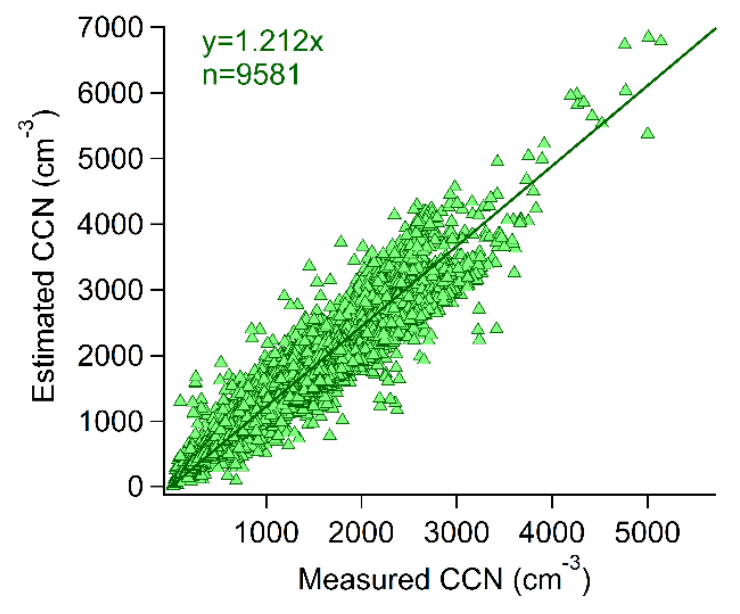

(d) 

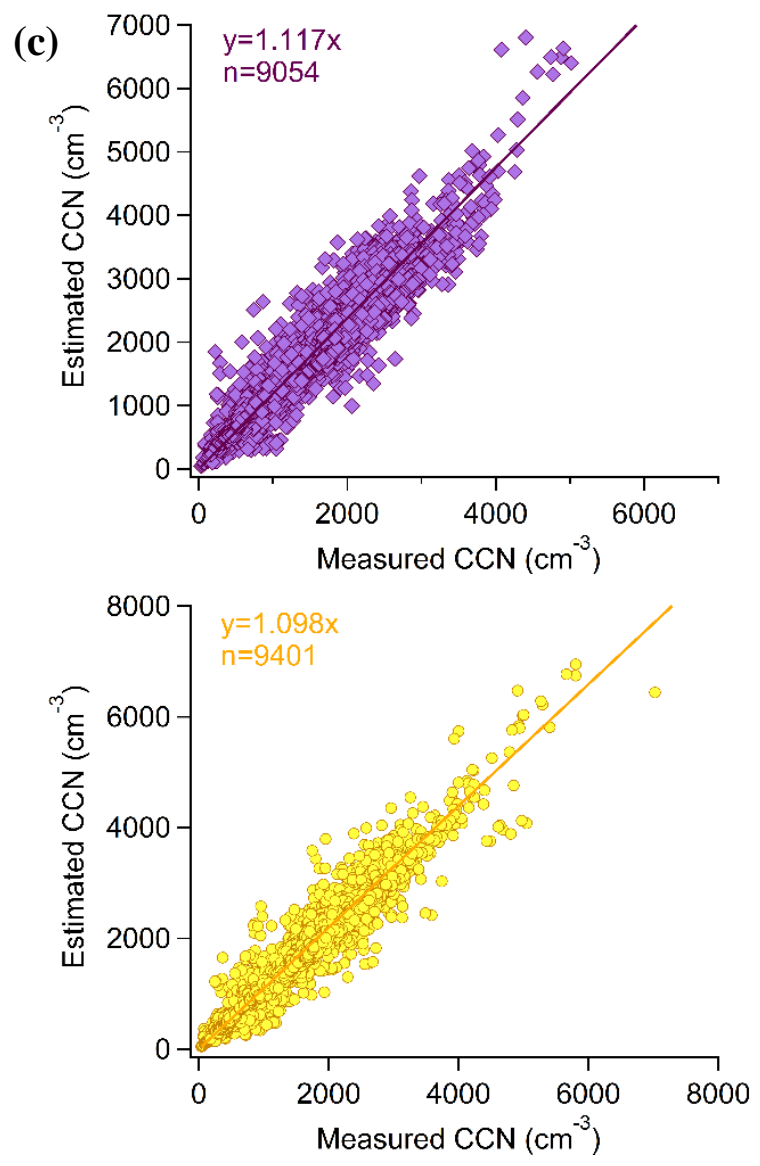

Figure S2. Measured against estimated CCN concentrations at each supersaturation $s$ (a) $0.38 \%$, (b) $0.52 \%$, (c) $0.66 \%$, and (d) $0.73 \%$. 
(a)
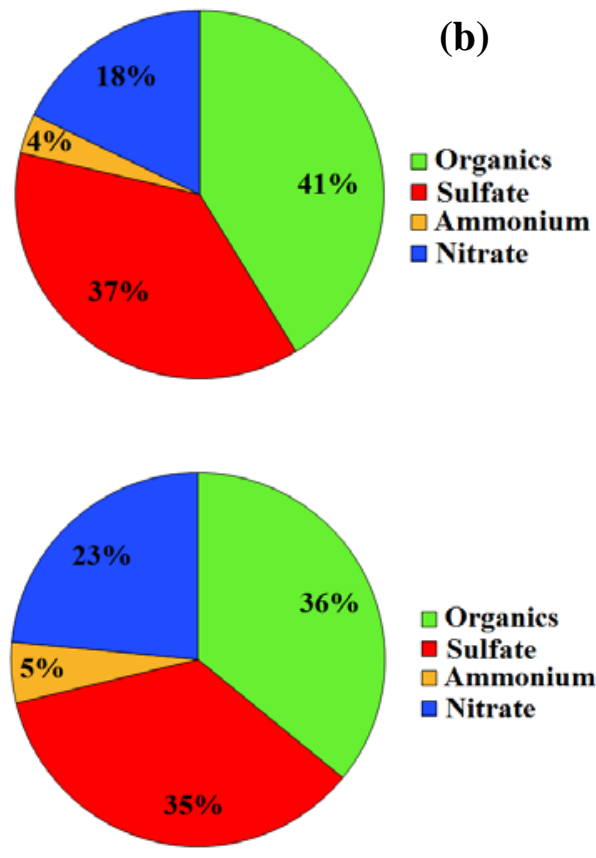

$\square$ Organic

- Sulfate

$\square$ Ammonium

$\square$ Nitrate

(c)
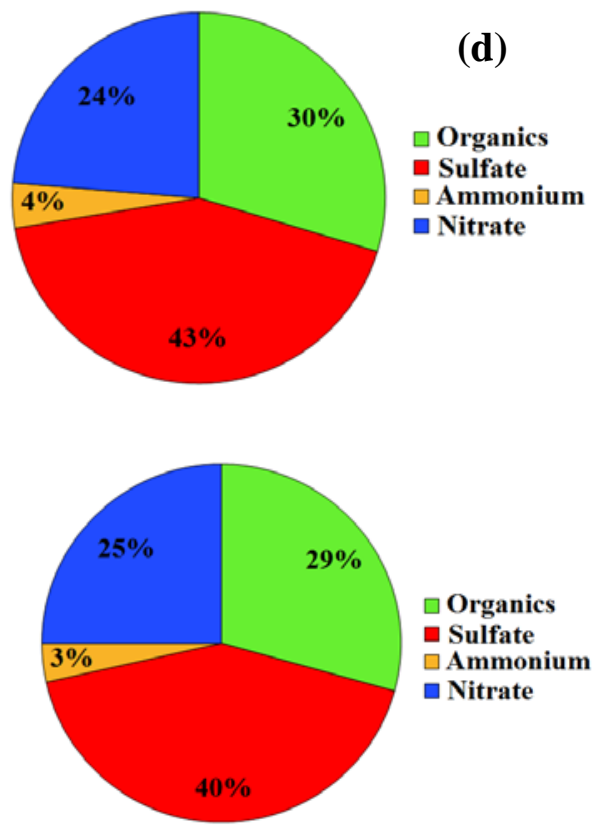
(e)
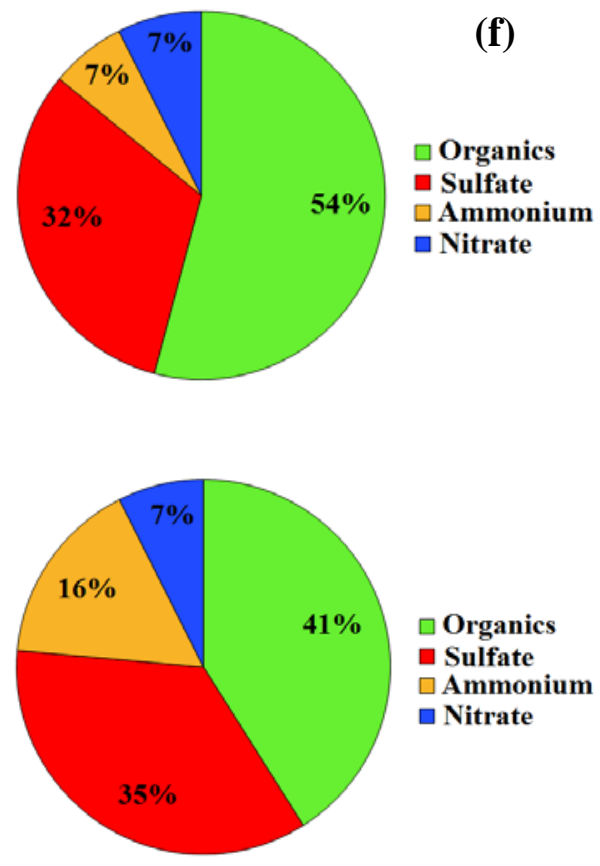

(g)
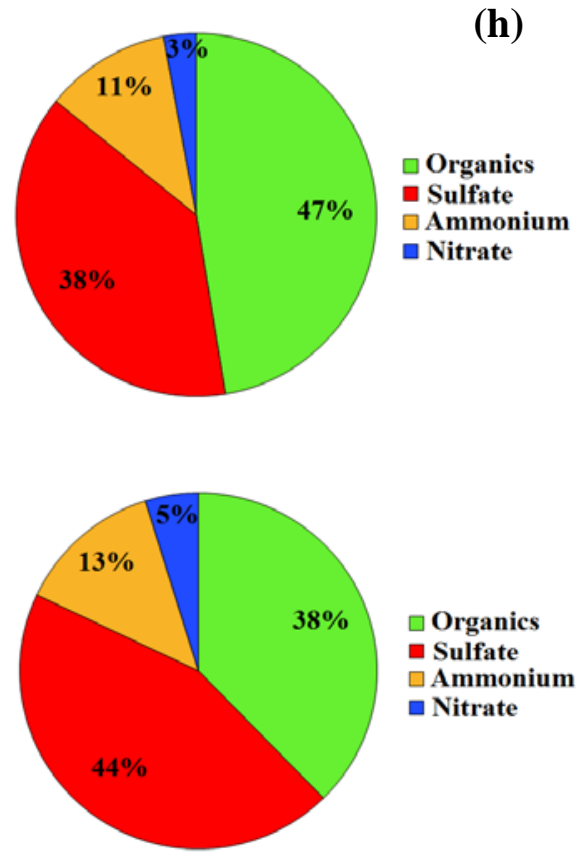

Figure S3. Seasonal evolution of the chemical composition (organics, sulfate, ammonium, and nitrate) of the total estimated $\mathrm{PM}_{1}$ mass throughout (a) winter, (b) spring, (c) summer, and (d) autumn from June 2008 to December 2011 as derived from the bulk $\mathrm{PM}_{10}$ 24-h quartz fiber filters, and of the non-refractory submicron aerosol particles during (e) winter, (f) spring, (g) summer, and (h) autumn from May 2012 to May 2015 as derived from the ACSM measurements. 


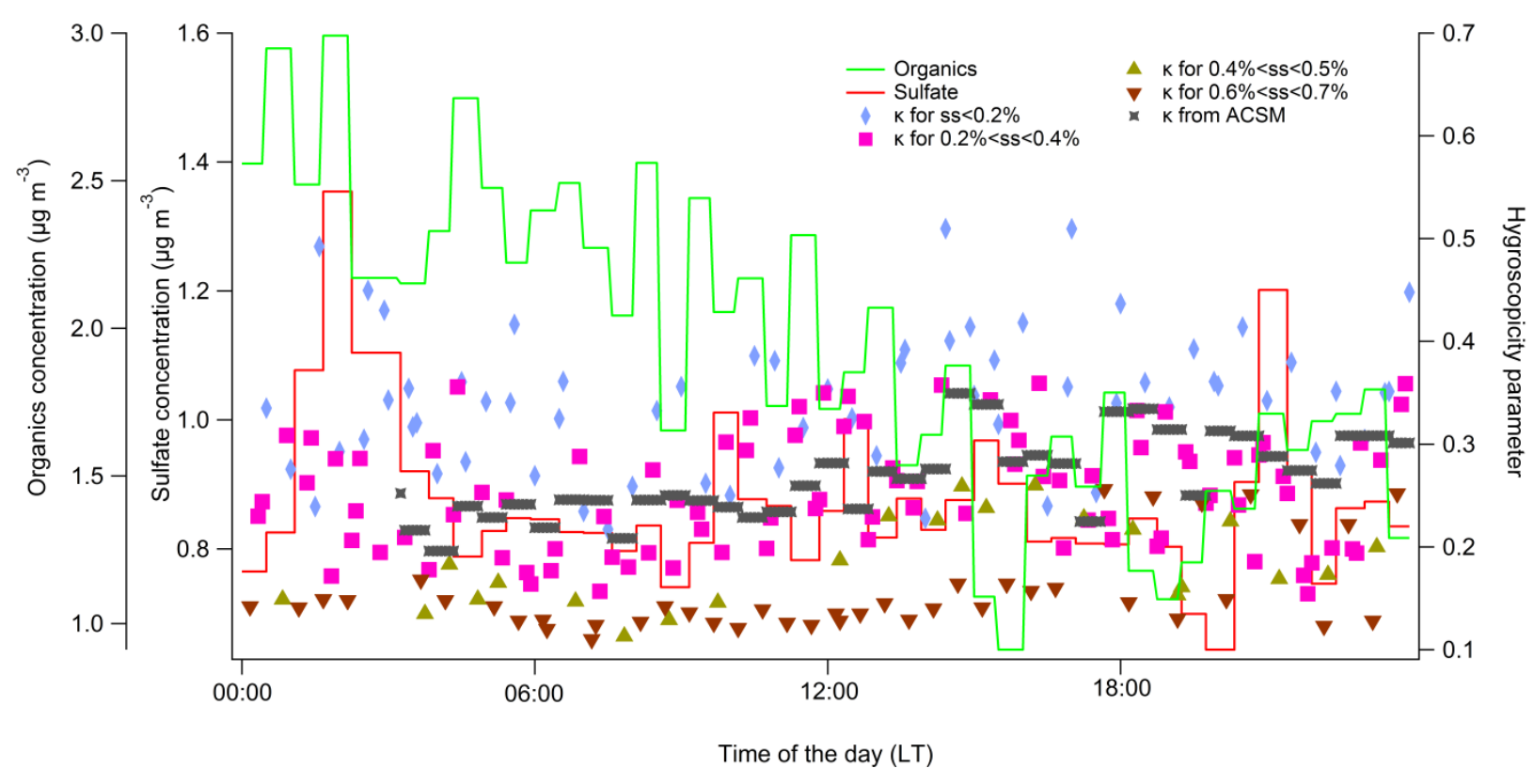

Figure S4. Diurnal evolution of organic and sulfate loadings together with the hygroscopicity parameter $(\kappa)$ derived from ACSM data (grey cross) and the $\kappa$ obtained from the CCN counter for critical supersaturation under $0.20 \%$ (blue rhombus), from 0.20 to $0.40 \%$ (magenta square), from 0.40 to $0.50 \%$ (green triangle), and from 0.60 to $0.73 \%$ (brown triangle), respectively on 29 August 2012. 
Table S1. Regression statistics for the CCN closure.

\begin{tabular}{|c|c|c|}
\hline $\begin{array}{c}\text { Supersaturation, } s(\%) \\
(\mathrm{n}=\text { data points) }\end{array}$ & $\frac{\mathrm{CCN}_{\text {estimated }}}{\mathrm{CCN}_{\text {measured }}}$ & $\mathrm{R}^{2}$ \\
\hline $0.38(\mathrm{n}=9167)$ & 1.221 & 0.885 \\
\hline $0.52(\mathrm{n}=9581)$ & 1.212 & 0.892 \\
\hline $0.66(\mathrm{n}=9054)$ & 1.117 & 0.911 \\
\hline $0.73(\mathrm{n}=9401)$ & 1.098 & 0.933 \\
\hline
\end{tabular}

Table S2. Seasonal wind direction, starting time of a NPF event ( $\left.t_{\text {start }}\right)$, decoupling time $\left(t_{d e c}\right)$, $R_{\mathrm{s}}$ before (bef) being the time step between $t_{\text {start }}$ and $t_{d e c}$, and after (aft) being the time step between $t_{d e c}$ and the end of CCN production, and the increase of the $R_{s}(\%)$ observed after the $t_{d e c}$ for supersaturations $0.10,0.38,0.52,0.66,0.73$, and $1.00 \%$, respectively. Time is in LT.

\begin{tabular}{|c|c|c|c|c|c|c|c|c|c|c|c|c|c|c|c|c|c|c|c|c|c|c|}
\hline & Date & $\begin{array}{c}\text { Dir } \\
\left(^{o}\right)\end{array}$ & $\begin{array}{c}t_{\text {start }} \\
(L T)\end{array}$ & $\begin{array}{c}t_{d e t} \\
(L T)\end{array}$ & $\begin{array}{l}R_{0.10} \\
\text { (bef) }\end{array}$ & $\begin{array}{l}\text { Ro.10 } \\
\text { (aft) }\end{array}$ & $\begin{array}{c}\text { Increase } \\
\text { of the } \\
R_{0.10}(\%)\end{array}$ & $\begin{array}{l}\text { Ro.38 } \\
\text { (bef) }\end{array}$ & $\begin{array}{l}\text { Ro.38 } \\
\text { (aft) }\end{array}$ & $\begin{array}{c}\text { Increase } \\
\text { of the } \\
\text { Ro.38 (\%) }^{2}(\%)\end{array}$ & $\begin{array}{l}\text { Ro.52 } \\
\text { (bef) }\end{array}$ & $\begin{array}{l}\text { Ro.52 } \\
\text { (aft) }\end{array}$ & $\begin{array}{c}\text { Increase } \\
\text { of the } \\
R_{0.52}(\%)\end{array}$ & $\begin{array}{l}\text { Ro.66 } \\
\text { (bef) }\end{array}$ & $\begin{array}{l}\text { Ro.66 } \\
\text { (aft) }\end{array}$ & $\begin{array}{c}\text { Increase } \\
\text { of the } \\
R_{0.66}(\%)\end{array}$ & $\begin{array}{l}\text { Ro.73 } \\
\text { (bef) }\end{array}$ & $\begin{array}{l}\text { Ro.73 } \\
\text { (aft) }\end{array}$ & $\begin{array}{c}\text { Increase } \\
\text { of the } \\
R_{0.73(\%)}\end{array}$ & $\begin{array}{l}R_{1.00} \\
\text { (bef) }\end{array}$ & $\begin{array}{l}\text { R1.00 } \\
\text { (aft) }\end{array}$ & $\begin{array}{c}\text { Increase } \\
\text { of the } \\
R_{1.00}(\%)\end{array}$ \\
\hline \multicolumn{23}{|c|}{ Winter } \\
\hline 1 & $4 / 12 / 2008$ & $\mathrm{~S} / \mathrm{SW}$ & $11: 10$ & $12: 05$ & 1.17 & 1.33 & 14 & 1.12 & 1.28 & 14 & 1.09 & 1.23 & 13 & 1.09 & 1.22 & 12 & 1.08 & 1.22 & 13 & 1.07 & 1.26 & 18 \\
\hline 2 & $13 / 12 / 2008$ & $\mathrm{~W}$ & $10: 25$ & $12: 55$ & 0.99 & 1.42 & 43 & 1.05 & 2.22 & 111 & 1.08 & 2.28 & 111 & 1.10 & 2.21 & 101 & 1.10 & 2.17 & 97 & 1.10 & 2.07 & 88 \\
\hline 3 & $26 / 12 / 2009$ & S/SW & $12: 20$ & $14: 55$ & 2.87 & 4.93 & 72 & 2.62 & 5.38 & 105 & 2.49 & 5.27 & 112 & 2.42 & 5.24 & 117 & 2.39 & 5.26 & 120 & 2.29 & 5.61 & 145 \\
\hline 4 & $19 / 2 / 2010$ & W & $7: 45$ & $16: 15$ & 1.17 & 1.43 & 22 & 1.40 & 2.31 & 65 & 1.42 & 2.20 & 55 & 1.38 & 2.04 & 48 & 1.32 & 1.91 & 45 & 1.32 & 2.00 & 52 \\
\hline 5 & $26 / 2 / 2010$ & NW & $11: 30$ & $14: 10$ & 1.18 & 1.34 & 14 & 1.52 & 2.09 & 38 & 1.58 & 2.15 & 36 & 1.58 & 2.12 & 34 & 1.58 & 2.11 & 34 & 1.55 & 2.03 & 31 \\
\hline 6 & $3 / 1 / 2011$ & S/SW & $14: 05$ & $15: 55$ & 1.19 & 1.52 & 28 & 1.41 & 1.92 & 36 & 1.40 & 1.90 & 36 & 1.40 & 1.79 & 28 & 1.40 & 1.84 & 31 & 1.41 & 1.76 & 25 \\
\hline 7 & $6 / 1 / 2011$ & $\mathrm{~N}$ & $14: 45$ & $15: 35$ & 0.97 & 1.05 & 8 & 1.04 & 1.10 & 6 & 1.07 & 1.17 & 9 & 1.06 & 1.14 & 8 & 1.06 & 1.13 & 7 & 1.07 & 1.11 & 4 \\
\hline 8 & $17 / 1 / 2011$ & NE & $11: 10$ & $14: 20$ & 0.95 & 0.83 & -13 & 0.95 & 0.96 & 1 & 0.97 & 1.17 & 21 & 0.98 & 1.44 & 47 & 1.00 & 1.82 & 82 & 1.03 & 2.57 & 150 \\
\hline 9 & $19 / 1 / 2011$ & $\mathrm{~N}$ & $12: 45$ & $17: 40$ & 0.64 & 1.40 & 119 & 0.84 & 1.81 & 115 & 0.85 & 1.73 & 104 & 0.86 & 1.66 & 93 & 0.87 & 1.59 & 83 & 0.89 & 1.59 & 79 \\
\hline
\end{tabular}




\begin{tabular}{|c|c|c|c|c|c|c|}
\hline 10 & $1 / 2 / 2011$ & $\mathrm{~N}$ & $11: 00$ & $17: 00$ & 0.83 & 0.63 \\
\hline 11 & $7 / 2 / 2011$ & $\mathrm{E}$ & $13: 50$ & $14: 40$ & 1.29 & 2.19 \\
\hline 12 & $10 / 2 / 2011$ & $\mathrm{~N}$ & $11: 30$ & $16: 30$ & 0.66 & 0.70 \\
\hline 13 & $11 / 2 / 2011$ & $\mathrm{~N}$ & $10: 05$ & $17: 15$ & 0.70 & 1.00 \\
\hline 14 & $1 / 1 / 2012$ & $\mathrm{NE}$ & $5: 45$ & - & - & - \\
\hline 15 & $26 / 1 / 2012$ & $\mathrm{~W}$ & $4: 50$ & - & - & - \\
\hline 16 & $21 / 1 / 2013$ & $\mathrm{E}$ & $11: 00$ & $11: 50$ & 1.07 & 1.31 \\
\hline 17 & $23 / 1 / 2013$ & $\mathrm{~W}$ & $10: 45$ & $12: 10$ & 0.91 & 0.97 \\
\hline 18 & $8 / 1 / 2014$ & $\mathrm{NE}$ & $13: 10$ & $14: 30$ & 1.18 & 1.31 \\
\hline 19 & $10 / 1 / 2014$ & $\mathrm{NW}$ & $11: 30$ & $12: 50$ & 0.81 & 0.89 \\
\hline 20 & $23 / 1 / 2014$ & $\mathrm{~W}$ & $10: 05$ & $11: 25$ & 1.09 & 1.43 \\
\hline 21 & $30 / 1 / 2014$ & $\mathrm{E}$ & $11: 05$ & $12: 15$ & 0.89 & 0.94 \\
\hline 22 & $22 / 12 / 2014$ & $\mathrm{~N}$ & $9: 20$ & $12: 30$ & 0.95 & 0.91 \\
\hline 23 & $11 / 1 / 2015$ & $\mathrm{NW}$ & $11: 25$ & $11: 50$ & 0.90 & 0.99 \\
\hline 24 & $23 / 1 / 2015$ & $\mathrm{~S} / \mathrm{SW}$ & $9: 20$ & $10: 50$ & 0.78 & 1.11 \\
\hline Spring & \multicolumn{5}{|c|}{} \\
\hline 25 & $14 / 3 / 2009$ & $\mathrm{NW}$ & $10: 15$ & $12: 30$ & 1.04 & 1.25 \\
\hline 26 & $19 / 3 / 2009$ & $\mathrm{~N}$ & $8: 50$ & $14: 05$ & 1.01 & 1.09 \\
\hline 27 & $30 / 3 / 2009$ & $\mathrm{NW}$ & $10: 35$ & $14: 40$ & 0.95 & 1.27 \\
\hline 28 & $22 / 5 / 2009$ & $\mathrm{NE}$ & $9: 00$ & $10: 45$ & 0.99 & 1.08 \\
\hline 29 & $28 / 5 / 2009$ & $\mathrm{NE}$ & $8: 00$ & $8: 35$ & 1.07 & 1.46 \\
\hline 30 & $13 / 3 / 2010$ & $\mathrm{~W}$ & $10: 20$ & $11: 50$ & 1.02 & 1.05 \\
\hline 31 & $16 / 3 / 2010$ & $\mathrm{~N}$ & $10: 00$ & $12: 55$ & 0.89 & 0.88 \\
\hline 32 & $18 / 3 / 2010$ & $\mathrm{~N}$ & $11: 15$ & $14: 00$ & 0.95 & 0.97 \\
\hline 33 & $19 / 3 / 2010$ & $\mathrm{~N}$ & $13: 15$ & $16: 05$ & 1.12 & 1.19 \\
\hline 34 & $22 / 3 / 2010$ & $\mathrm{~W}$ & $10: 50$ & $12: 15$ & 1.02 & 1.06 \\
\hline 35 & $27 / 3 / 2010$ & $\mathrm{~N}$ & $10: 05$ & $12: 55$ & 0.81 & 1.10 \\
\hline 36 & $30 / 3 / 2010$ & $\mathrm{NW}$ & $11: 20$ & $11: 30$ & 1.00 & 1.10 \\
\hline 37 & $7 / 4 / 2010$ & $\mathrm{NW}$ & $9: 00$ & $12: 15$ & 1.54 & 1.61 \\
\hline 38 & $8 / 4 / 2010$ & $\mathrm{~N}$ & $11: 30$ & $13: 30$ & 0.93 & 1.30 \\
\hline 39 & $9 / 4 / 2010$ & $\mathrm{NE}$ & $10: 25$ & $12: 05$ & 1.16 & 1.21 \\
\hline 40 & $15 / 4 / 2010$ & $\mathrm{~S} / \mathrm{SW}$ & $13: 00$ & $14: 25$ & 0.90 & 0.89 \\
\hline 41 & $17 / 4 / 2010$ & $\mathrm{~S} / \mathrm{SW}$ & $9: 50$ & $13: 35$ & 1.53 & 2.32 \\
\hline 42 & $29 / 4 / 2010$ & $\mathrm{NE}$ & $10: 15$ & $13: 50$ & 0.81 & 1.17 \\
\hline 43 & $1 / 5 / 2010$ & $\mathrm{NE}$ & $9: 25$ & $10: 40$ & 1.00 & 1.18 \\
\hline
\end{tabular}

\begin{tabular}{|c|c|c|c|c|c|c|c|c|c|c|c|c|c|c|c|}
\hline-24 & 0.96 & 1.04 & 8 & 0.98 & 1.43 & 46 & 0.98 & 1.98 & 102 & 0.98 & 2.19 & 123 & 1.04 & 3.04 & 192 \\
\hline 70 & 1.45 & 2.58 & 78 & 1.41 & 2.52 & 79 & 1.38 & 2.59 & 88 & 1.38 & 2.64 & 91 & 1.45 & 2.95 & 103 \\
\hline 6 & 0.79 & 0.80 & 1 & 0.84 & 0.87 & 4 & 0.86 & 0.96 & 12 & 0.87 & 1.01 & 16 & 0.87 & 1.16 & 33 \\
\hline 43 & 0.68 & 1.19 & 75 & 0.66 & 1.13 & 71 & 0.65 & 1.04 & 60 & 0.64 & 0.99 & 55 & 0.64 & 1.11 & 73 \\
\hline- & - & - & - & - & - & - & - & - & - & - & - & - & - & - & - \\
\hline- & - & - & - & - & - & - & - & - & - & - & - & - & - & - & - \\
\hline 22 & 1.04 & 1.34 & 29 & 1.01 & 1.29 & 28 & 1.01 & 1.26 & 25 & 1.00 & 1.24 & 24 & 1.00 & 1.24 & 24 \\
\hline 7 & 1.13 & 1.42 & 26 & 1.12 & 1.36 & 21 & 1.12 & 1.33 & 19 & 1.10 & 1.32 & 20 & 1.11 & 1.28 & 15 \\
\hline 11 & 1.75 & 1.94 & 11 & 1.77 & 1.91 & 8 & 1.78 & 1.90 & 7 & 1.80 & 1.89 & 5 & 1.83 & 1.92 & 5 \\
\hline 10 & 1.14 & 1.41 & 24 & 1.11 & 1.33 & 20 & 1.11 & 1.30 & 17 & 1.10 & 1.29 & 17 & 1.10 & 1.33 & 21 \\
\hline 31 & 1.08 & 1.66 & 54 & 1.03 & 1.5 & 46 & 0.99 & 1.37 & 38 & 0.97 & 1.37 & 41 & 0.94 & 1.36 & 45 \\
\hline 6 & 1.12 & 1.33 & 19 & 1.09 & 1.27 & 17 & 1.09 & 1.24 & 14 & 1.08 & 1.21 & 12 & 1.07 & 1.19 & 11 \\
\hline-4 & 1.11 & 1.71 & 54 & 1.11 & 1.66 & 50 & 1.19 & 1.65 & 39 & 1.07 & 1.45 & 36 & 1.10 & 1.42 & 29 \\
\hline 10 & 1.37 & 2.03 & 48 & 1.49 & 2.02 & 36 & 1.63 & 2.14 & 31 & 1.69 & 2.14 & 27 & 1.77 & 2.08 & 18 \\
\hline 42 & 0.77 & 1.02 & 32 & 0.77 & 1.00 & 30 & 0.78 & 1.01 & 29 & 0.78 & 1.01 & 29 & 0.79 & 1.02 & 29 \\
\hline 20 & 0.93 & 2.20 & 137 & 0.93 & 3.32 & 257 & 0.93 & 3.69 & 297 & 0.93 & 4.01 & 331 & 0.95 & 3.84 & 304 \\
\hline 8 & 1.10 & 1.72 & 56 & 1.16 & 2.35 & 103 & 1.21 & 3.19 & 164 & 1.23 & 3.50 & 185 & 1.27 & 4.36 & 243 \\
\hline 34 & 0.90 & 1.02 & 13 & 0.90 & 1.01 & 12 & 0.89 & 0.99 & 11 & 0.89 & 0.98 & 10 & 0.88 & 0.97 & 10 \\
\hline 9 & 1.01 & 1.08 & 7 & 0.98 & 1.01 & 3 & 0.92 & 0.98 & 7 & 0.90 & 0.95 & 6 & 0.86 & 0.92 & 7 \\
\hline 36 & 1.14 & 1.46 & 28 & 1.12 & 1.36 & 21 & 1.09 & 1.30 & 19 & 1.08 & 1.30 & 20 & 1.05 & 1.35 & 29 \\
\hline 3 & 1.09 & 1.16 & 6 & 1.08 & 1.12 & 4 & 1.07 & 1.10 & 3 & 1.07 & 1.09 & 2 & 1.05 & 1.04 & -1 \\
\hline-1 & 0.98 & 1.03 & 5 & 0.99 & 1.06 & 7 & 0.99 & 1.04 & 5 & 0.99 & 1.03 & 4 & 0.99 & 1.00 & 1 \\
\hline 2 & 1.03 & 1.11 & 8 & 1.03 & 1.09 & 6 & 1.01 & 1.04 & 3 & 1.00 & 1.02 & 2 & 0.99 & 1.00 & 1 \\
\hline 6 & 1.20 & 1.23 & 3 & 1.20 & 1.27 & 6 & 1.20 & 1.28 & 7 & 1.20 & 1.28 & 7 & 1.18 & 1.25 & 6 \\
\hline 4 & 0.99 & 1.00 & 1 & 0.98 & 0.98 & 0 & 0.98 & 0.97 & -1 & 0.98 & 0.97 & -1 & 0.98 & 0.96 & -2 \\
\hline 36 & 1.03 & 1.40 & 36 & 1.08 & 1.47 & 36 & 1.09 & 1.40 & 28 & 1.11 & 1.39 & 25 & 1.11 & 1.41 & 27 \\
\hline 10 & 1.04 & 1.17 & 13 & 1.08 & 1.21 & 12 & 1.03 & 1.07 & 4 & 1.03 & 1.04 & 1 & 1.05 & 1.03 & -2 \\
\hline 5 & 1.81 & 1.76 & -3 & 1.75 & 2.04 & 17 & 1.73 & 2.35 & 36 & 1.71 & 2.46 & 44 & 1.66 & 2.95 & 78 \\
\hline 40 & 1.00 & 1.37 & 37 & 0.99 & 1.29 & 30 & 0.98 & 1.23 & 26 & 0.97 & 1.22 & 26 & 0.97 & 1.19 & 23 \\
\hline 4 & 1.24 & 1.52 & 23 & 1.24 & 1.49 & 20 & 1.23 & 1.46 & 19 & 1.29 & 1.48 & 15 & 1.28 & 1.49 & 16 \\
\hline-1 & 0.89 & 1.05 & 18 & 0.89 & 1.06 & 19 & 0.89 & 1.10 & 24 & 0.90 & 1.20 & 33 & 0.90 & 1.52 & 69 \\
\hline 52 & 2.12 & 3.44 & 62 & 2.21 & 3.47 & 57 & 2.16 & 3.32 & 54 & 2.14 & 3.24 & 51 & 2.13 & 3.26 & 53 \\
\hline 44 & 0.88 & 1.23 & 40 & 0.89 & 1.21 & 36 & 0.89 & 1.17 & 31 & 0.89 & 1.16 & 30 & 0.88 & 1.14 & 30 \\
\hline 18 & 1.00 & 1.02 & 2 & 1.00 & 1.00 & 0 & 0.98 & 0.99 & 1 & 0.97 & 0.98 & 1 & 0.93 & 0.95 & 2 \\
\hline
\end{tabular}




\begin{tabular}{|c|c|c|c|c|c|c|c|c|c|c|c|c|c|c|c|c|c|c|c|c|c|c|}
\hline 44 & $30 / 5 / 2010$ & $\mathrm{~N}$ & $7: 55$ & 9:40 & 0.87 & 0.83 & -5 & 0.87 & 0.97 & 11 & 0.88 & 1.04 & 18 & 0.89 & 1.09 & 22 & 0.89 & 1.09 & 22 & 0.89 & 1.12 & 26 \\
\hline 45 & $9 / 3 / 2011$ & $\mathrm{NE}$ & $10: 35$ & 11:50 & 1.01 & 1.26 & 25 & 1.10 & 1.41 & 28 & 1.11 & 1.50 & 35 & 1.11 & 1.48 & 33 & 1.11 & 1.47 & 32 & 1.08 & 1.43 & 32 \\
\hline 46 & $19 / 3 / 2011$ & $\mathrm{~W}$ & $10: 25$ & $12: 05$ & 0.78 & 1.00 & 28 & 0.91 & 1.04 & 14 & 0.90 & 1.01 & 12 & 0.89 & 0.97 & 9 & 0.89 & 0.97 & 9 & 0.87 & 0.96 & 10 \\
\hline 47 & $29 / 3 / 2011$ & S/SW & $11: 55$ & $13: 00$ & 1.03 & 1.25 & 21 & 1.04 & 1.34 & 29 & 1.05 & 1.37 & 30 & 1.06 & 1.40 & 32 & 1.07 & 1.43 & 34 & 1.07 & 1.48 & 38 \\
\hline 48 & $12 / 4 / 2011$ & $\mathrm{~N}$ & 11:00 & $13: 45$ & 1.07 & 1.60 & 50 & 1.05 & 1.69 & 61 & 1.05 & 1.58 & 50 & 1.05 & 1.54 & 47 & 1.06 & 1.50 & 42 & 1.05 & 1.44 & 37 \\
\hline 49 & $3 / 5 / 2011$ & S/SW & $9: 40$ & $12: 30$ & 0.92 & 1.06 & 15 & 1.08 & 1.36 & 26 & 1.07 & 1.33 & 24 & 1.07 & 1.27 & 19 & 1.09 & 1.27 & 17 & 1.08 & 1.21 & 12 \\
\hline 50 & 4/5/2011 & W & $10: 30$ & $13: 50$ & 1.42 & 3.87 & 173 & 2.12 & 4.01 & 89 & 2.18 & 3.86 & 77 & 2.17 & 3.55 & 64 & 2.15 & 3.43 & 60 & 2.11 & 2.99 & 42 \\
\hline 51 & $14 / 5 / 2011$ & $\mathrm{NE}$ & 9:25 & $10: 50$ & 0.96 & 1.00 & 4 & 1.03 & 1.08 & 5 & 1.00 & 1.03 & 3 & 0.94 & 1.02 & 9 & 0.92 & 1.00 & 9 & 0.88 & 0.96 & 9 \\
\hline 52 & $25 / 4 / 2012$ & S/SW & $12: 55$ & - & - & - & - & - & - & - & - & - & - & - & - & - & - & - & - & - & - & - \\
\hline 53 & $1 / 5 / 2012$ & $\mathrm{NE}$ & 9:10 & $12: 05$ & 1.12 & 1.21 & 8 & 1.18 & 1.34 & 14 & 1.17 & 1.31 & 12 & 1.17 & 1.25 & 7 & 1.19 & 1.26 & 6 & 1.18 & 1.23 & 4 \\
\hline 54 & $14 / 5 / 2012$ & $\mathrm{~N}$ & $8: 45$ & $10: 55$ & 1.03 & 1.10 & 7 & 1.11 & 1.21 & 9 & 1.08 & 1.14 & 6 & 1.02 & 1.21 & 19 & 1.00 & 1.16 & 16 & 1.06 & 1.12 & 6 \\
\hline 55 & $6 / 3 / 2013$ & $\mathrm{~N}$ & $10: 45$ & 12:00 & 1.02 & 1.09 & 7 & 1.13 & 1.44 & 27 & 1.14 & 1.52 & 33 & 1.14 & 1.51 & 32 & 1.14 & 1.50 & 32 & 1.11 & 1.46 & 32 \\
\hline 56 & $10 / 3 / 2013$ & $\mathrm{~W}$ & 9:35 & $11: 45$ & 1.26 & 3.25 & 158 & 1.18 & 3.59 & 204 & 1.21 & 3.30 & 173 & 1.20 & 3.07 & 156 & 1.19 & 2.71 & 128 & 1.17 & 2.40 & 105 \\
\hline 57 & $13 / 3 / 2013$ & $\mathrm{~W}$ & 9:45 & $13: 15$ & 1.20 & 2.36 & 97 & 1.48 & 3.49 & 136 & 1.53 & 3.20 & 109 & 1.43 & 2.79 & 95 & 1.49 & 2.74 & 84 & 1.46 & 2.50 & 71 \\
\hline 58 & $23 / 3 / 2013$ & NW & $8: 40$ & $14: 50$ & 0.59 & 0.67 & 14 & 0.54 & 0.77 & 43 & 0.54 & 0.77 & 43 & 0.56 & 0.78 & 39 & 0.57 & 0.85 & 49 & 0.59 & 1.05 & 78 \\
\hline 59 & $24 / 3 / 2013$ & NW & $7: 40$ & 9:30 & 0.95 & 2.07 & 118 & 1.05 & 1.84 & 75 & 1.10 & 1.85 & 68 & 1.07 & 1.71 & 60 & 1.08 & 1.71 & 58 & 1.13 & 1.71 & 51 \\
\hline 60 & $27 / 3 / 2013$ & $\mathrm{~W}$ & 9:10 & $13: 50$ & 1.26 & 1.51 & 20 & 1.35 & 1.80 & 33 & 1.37 & 1.78 & 30 & 1.36 & 1.71 & 26 & 1.36 & 1.68 & 24 & 1.37 & 1.67 & 22 \\
\hline 61 & $5 / 4 / 2013$ & S/SW & $10: 40$ & $12: 20$ & 1.62 & 1.95 & 20 & 1.47 & 2.04 & 39 & 1.46 & 1.92 & 32 & 1.48 & 1.99 & 34 & 1.44 & 1.91 & 33 & 1.43 & 1.96 & 37 \\
\hline 62 & $19 / 4 / 2013$ & $\mathrm{NE}$ & 11:00 & $13: 10$ & 1.07 & 1.72 & 61 & 1.41 & 2.19 & 55 & 1.39 & 2.05 & 47 & 1.45 & 1.97 & 36 & 1.38 & 1.88 & 36 & 1.36 & 1.85 & 36 \\
\hline 63 & $21 / 4 / 2013$ & $\mathrm{NE}$ & $12: 10$ & $15: 00$ & 1.04 & 1.40 & 35 & 1.12 & 1.54 & 38 & 1.12 & 1.50 & 34 & 1.06 & 1.45 & 37 & 1.06 & 1.45 & 37 & 1.06 & 1.51 & 42 \\
\hline 64 & $1 / 5 / 2013$ & $\mathrm{NE}$ & $10: 35$ & $11: 45$ & 0.98 & 1.06 & 8 & 1.00 & 1.08 & 8 & 1.01 & 1.05 & 4 & 1.00 & 1.01 & 1 & 1.00 & 1.01 & 1 & 0.99 & 1.00 & 1 \\
\hline 65 & $22 / 5 / 2013$ & NW & $12: 15$ & $15: 30$ & 0.90 & 1.12 & 24 & 0.92 & 1.14 & 24 & 0.94 & 1.11 & 18 & 0.99 & 1.11 & 12 & 1.02 & 1.11 & 9 & 1.08 & 1.15 & 6 \\
\hline 66 & $13 / 3 / 2014$ & $\mathrm{NE}$ & $12: 35$ & $20: 05$ & 1.08 & 1.35 & 25 & 1.01 & 1.17 & 16 & 0.99 & 1.19 & 20 & 0.99 & 1.30 & 31 & 0.99 & 1.36 & 37 & 1.00 & 1.52 & 52 \\
\hline 67 & $21 / 3 / 2014$ & $\mathrm{~N}$ & $10: 00$ & $21: 05$ & 0.92 & 1.09 & 18 & 0.76 & 1.96 & 158 & 0.75 & 2.12 & 183 & 0.75 & 2.20 & 193 & 0.75 & 2.21 & 195 & 0.75 & 2.21 & 195 \\
\hline 68 & $23 / 3 / 2014$ & $\mathrm{~W}$ & $8: 15$ & $10: 15$ & 1.05 & 1.31 & 25 & 1.19 & 1.37 & 15 & 1.19 & 1.31 & 10 & 1.19 & 1.27 & 7 & 1.19 & 1.26 & 6 & 1.22 & 1.26 & 3 \\
\hline 69 & $30 / 3 / 2014$ & $\mathrm{~N}$ & $12: 40$ & $13: 05$ & 0.37 & 0.80 & 116 & 0.38 & 0.81 & 113 & 0.41 & 0.82 & 100 & 0.46 & 0.83 & 80 & 0.47 & 0.84 & 79 & 0.53 & 0.85 & 60 \\
\hline 70 & $2 / 5 / 2014$ & S/SW & 10:40 & $13: 35$ & 1.01 & 1.19 & 18 & 1.12 & 1.40 & 25 & 1.10 & 1.36 & 24 & 1.10 & 1.30 & 18 & 1.11 & 1.29 & 16 & 1.11 & 1.24 & 12 \\
\hline 71 & $21 / 5 / 2014$ & $\mathrm{~N}$ & $8: 45$ & $10: 15$ & 0.99 & 1.03 & 4 & 1.05 & 1.13 & 8 & 1.06 & 1.14 & 8 & 1.07 & 1.10 & 3 & 1.06 & 1.08 & 2 & 1.05 & 1.09 & 4 \\
\hline 72 & $23 / 5 / 2014$ & $\mathrm{NE}$ & $8: 05$ & $12: 30$ & 0.82 & 0.97 & 18 & 0.94 & 1.05 & 12 & 0.92 & 1.05 & 14 & 0.91 & 1.05 & 15 & 0.92 & 1.05 & 14 & 1.05 & 1.06 & 1 \\
\hline 73 & $25 / 5 / 2014$ & NW & 9:40 & $11: 25$ & 0.84 & 0.95 & 13 & 0.98 & 1.14 & 16 & 0.97 & 1.13 & 16 & 0.95 & 1.14 & 20 & 0.95 & 1.15 & 21 & 0.95 & 1.13 & 19 \\
\hline 74 & $26 / 5 / 2014$ & S/SW & $9: 15$ & $11: 40$ & 1.02 & 1.19 & 17 & 0.97 & 1.20 & 24 & 0.96 & 1.20 & 25 & 1.02 & 1.21 & 19 & 1.02 & 1.19 & 17 & 1.17 & 1.23 & 5 \\
\hline 75 & $30 / 4 / 2015$ & $\mathrm{~W}$ & $7: 15$ & $9: 35$ & 0.97 & 1.69 & 74 & 1.12 & 3.00 & 168 & 1.08 & 3.06 & 183 & 1.07 & 3.11 & 191 & 1.08 & 3.16 & 193 & 1.12 & 3.27 & 192 \\
\hline 76 & $2 / 5 / 2015$ & NW & $8: 55$ & $10: 15$ & 0.99 & 1.15 & 16 & 1.04 & 1.29 & 24 & 1.07 & 1.31 & 22 & 1.06 & 1.32 & 25 & 1.06 & 1.36 & 28 & 1.07 & 1.50 & 40 \\
\hline \multicolumn{23}{|c|}{ Summer } \\
\hline 77 & $9 / 6 / 2008$ & $\mathrm{~W}$ & 9:45 & $14: 45$ & 1.50 & 3.56 & 137 & 1.72 & 2.42 & 41 & 1.69 & 2.24 & 33 & 1.68 & 2.12 & 26 & 1.68 & 2.09 & 24 & 1.70 & 2.02 & 19 \\
\hline
\end{tabular}




\begin{tabular}{|c|c|c|c|c|c|c|}
\hline 78 & $15 / 6 / 2008$ & NW & $7: 40$ & $9: 50$ & 0.92 & 0.98 \\
\hline 79 & $16 / 6 / 2008$ & N & $8: 40$ & $10: 20$ & 1.08 & 2.63 \\
\hline 80 & $20 / 6 / 2008$ & NW & $7: 25$ & $12: 05$ & 1.98 & 6.05 \\
\hline 81 & $24 / 7 / 2008$ & NW & $8: 45$ & $9: 30$ & 1.01 & 0.65 \\
\hline 82 & $25 / 7 / 2008$ & NW & $7: 50$ & $11: 00$ & 0.97 & 1.14 \\
\hline 83 & $26 / 7 / 2008$ & NW & $6: 50$ & $9: 50$ & 1.05 & 1.86 \\
\hline 84 & $30 / 7 / 2008$ & N & $9: 55$ & $12: 40$ & 1.08 & 1.21 \\
\hline 85 & $15 / 6 / 2009$ & NE & $10: 35$ & $14: 10$ & 0.95 & 1.48 \\
\hline 86 & $19 / 6 / 2009$ & NE & $11: 05$ & $13: 45$ & 0.99 & 1.10 \\
\hline 87 & $21 / 6 / 2009$ & S/SW & $10: 00$ & $12: 45$ & 0.91 & 1.18 \\
\hline 88 & $22 / 7 / 2009$ & N & $8: 50$ & $14: 25$ & 0.81 & 0.96 \\
\hline 89 & $29 / 7 / 2009$ & N & $10: 55$ & $12: 05$ & 0.97 & 1.18 \\
\hline 90 & $7 / 6 / 2010$ & N & $11: 05$ & $12: 20$ & 1.03 & 1.29 \\
\hline 91 & $5 / 7 / 2010$ & N & $9: 10$ & $15: 15$ & 1.03 & 1.22 \\
\hline 92 & $24 / 7 / 2010$ & N & $9: 00$ & $10: 35$ & 1.01 & 1.12 \\
\hline 93 & $18 / 8 / 2010$ & NW & $8: 20$ & $9: 55$ & 1.39 & 2.37 \\
\hline 94 & $30 / 8 / 2010$ & W & $10: 40$ & $12: 30$ & 1.27 & 1.72 \\
\hline 95 & $27 / 6 / 2011$ & N & $10: 15$ & $12: 40$ & 1.01 & 1.36 \\
\hline 96 & $11 / 7 / 2011$ & NE & $9: 45$ & $10: 55$ & 1.05 & 1.27 \\
\hline 97 & $16 / 7 / 2011$ & NE & $8: 25$ & $10: 55$ & 1.09 & 1.38 \\
\hline 98 & $18 / 7 / 2011$ & NE & $8: 20$ & $9: 55$ & 0.93 & 0.95 \\
\hline 99 & $22 / 7 / 2011$ & NW & $7: 15$ & $9: 05$ & 0.84 & 0.93 \\
\hline 100 & $26 / 7 / 2011$ & W & $8: 45$ & $13: 20$ & 0.85 & 1.35 \\
\hline 101 & $14 / 6 / 2012$ & W & $9: 25$ & $11: 25$ & 1.06 & 1.81 \\
\hline 102 & $16 / 6 / 2012$ & NE & $8: 50$ & $9: 40$ & 0.92 & 0.89 \\
\hline 103 & $17 / 6 / 2012$ & S/SW & $8: 25$ & $15: 20$ & 0.93 & 0.78 \\
\hline 104 & $18 / 6 / 2012$ & NE & $8: 20$ & $10: 25$ & 1.04 & 1.40 \\
\hline 105 & $19 / 6 / 2012$ & N & $11: 00$ & $12: 40$ & 1.08 & 1.14 \\
\hline 106 & $24 / 6 / 2012$ & NE & $8: 55$ & $11: 15$ & 1.01 & 1.06 \\
\hline 107 & $27 / 6 / 2012$ & N & $6: 45$ & $9: 50$ & 0.98 & 1.29 \\
\hline 108 & $29 / 6 / 2012$ & NE & $9: 50$ & $10: 30$ & 1.03 & 0.92 \\
\hline 109 & $19 / 7 / 2012$ & $\mathrm{~W}$ & $9: 05$ & $10: 45$ & 0.92 & 1.07 \\
\hline 110 & $31 / 7 / 2012$ & NE & $8: 40$ & $10: 05$ & 0.86 & 1.03 \\
\hline 111 & $1 / 8 / 2012$ & NE & $8: 30$ & $9: 10$ & 0.99 & 1.21 \\
\hline 112 & $19 / 8 / 2012$ & N & $9: 15$ & $13: 45$ & 0.78 & 0.87 \\
\hline & & & & & & \\
\hline
\end{tabular}
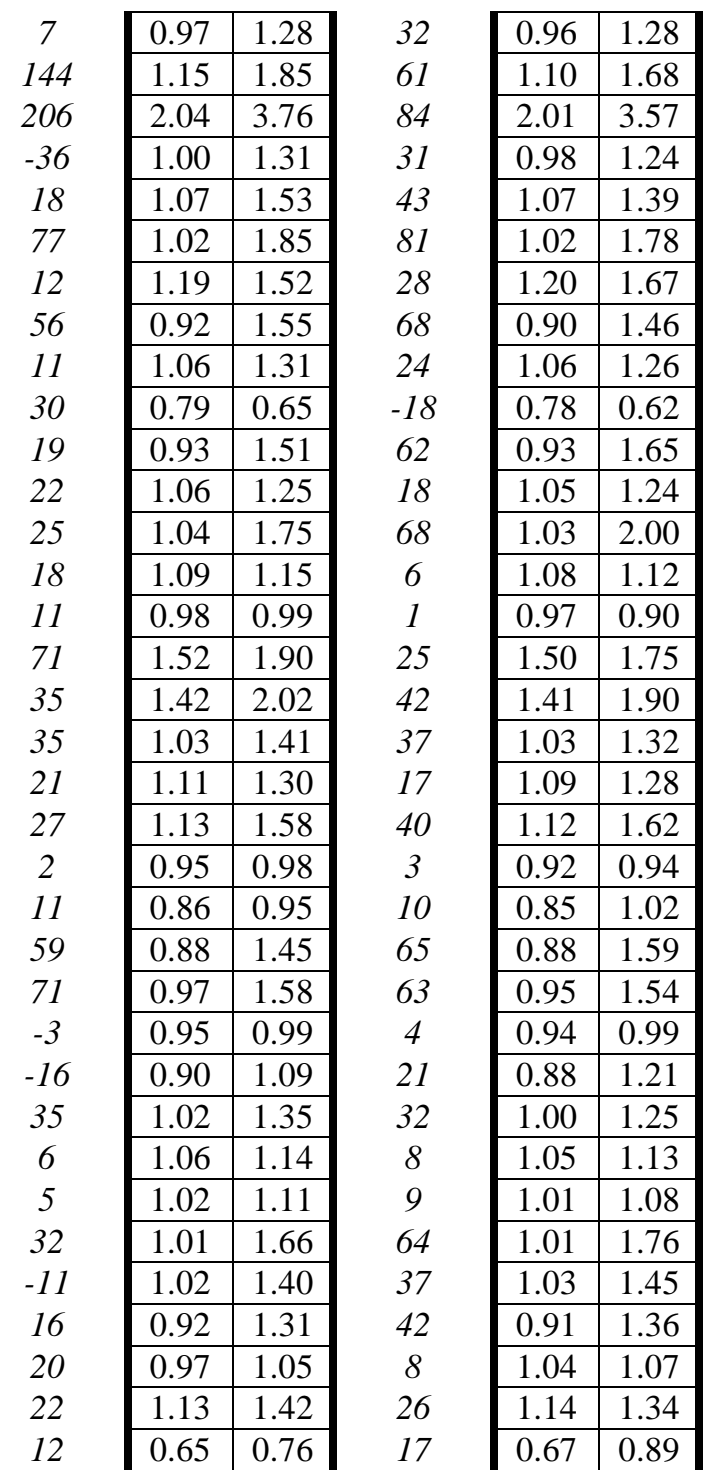

\begin{tabular}{c|c|c|}
33 & 0.96 & 1.24 \\
53 & 1.09 & 1.63 \\
78 & 1.98 & 3.44 \\
27 & 0.97 & 1.23 \\
30 & 1.07 & 1.29 \\
75 & 1.02 & 1.76 \\
39 & 1.20 & 1.80 \\
62 & 0.89 & 1.39 \\
19 & 1.03 & 1.25 \\
-21 & 0.77 & 0.61 \\
77 & 0.92 & 1.76 \\
18 & 1.02 & 1.24 \\
94 & 1.01 & 2.17 \\
4 & 1.08 & 1.10 \\
\hline 7 & 0.96 & 0.89 \\
17 & 1.46 & 1.62 \\
35 & 1.43 & 1.96 \\
28 & 1.02 & 1.28 \\
17 & 1.08 & 1.29 \\
\hline 45 & 1.10 & 1.68 \\
\hline 2 & 0.92 & 0.93 \\
20 & 0.86 & 1.07 \\
\hline 81 & 0.87 & 1.70 \\
\hline 62 & 0.93 & 1.51 \\
\hline 5 & 0.94 & 1.02 \\
\hline 38 & 0.87 & 1.29 \\
\hline 25 & 1.00 & 1.19 \\
8 & 1.04 & 1.11 \\
\hline 7 & 1.00 & 1.06 \\
74 & 1.02 & 1.83 \\
41 & 1.01 & 1.40 \\
49 & 0.91 & 1.48 \\
3 & 1.03 & 1.13 \\
18 & 1.13 & 1.32 \\
33 & 0.69 & 0.98 \\
\hline
\end{tabular}

\begin{tabular}{c|c|c|}
29 & 0.96 & 1.23 \\
50 & 1.09 & 1.61 \\
\hline 74 & 1.98 & 3.43 \\
27 & 0.97 & 1.24 \\
\hline 21 & 1.07 & 1.26 \\
\hline 73 & 1.02 & 1.76 \\
50 & 1.20 & 1.89 \\
56 & 0.88 & 1.38 \\
\hline 21 & 1.02 & 1.26 \\
-21 & 0.77 & 0.61 \\
91 & 0.92 & 1.81 \\
\hline 22 & 1.02 & 1.25 \\
115 & 1.01 & 2.21 \\
\hline 2 & 1.07 & 1.09 \\
\hline 7 & 0.96 & 0.88 \\
11 & 1.43 & 1.56 \\
\hline 37 & 1.39 & 1.88 \\
\hline 25 & 1.01 & 1.26 \\
\hline 19 & 1.07 & 1.29 \\
\hline 53 & 1.10 & 1.71 \\
\hline 1 & 0.91 & 0.93 \\
\hline 24 & 0.86 & 1.05 \\
\hline 95 & 0.87 & 1.74 \\
\hline 62 & 0.94 & 1.53 \\
9 & 0.94 & 1.03 \\
\hline 48 & 0.87 & 1.33 \\
\hline 19 & 0.99 & 1.17 \\
\hline 7 & 1.04 & 1.10 \\
\hline 6 & 1.00 & 1.06 \\
\hline 79 & 1.02 & 1.85 \\
39 & 1.01 & 1.40 \\
\hline 63 & 0.90 & 1.52 \\
10 & 1.02 & 1.16 \\
17 & 1.13 & 1.32 \\
42 & 0.69 & 1.06 \\
\hline
\end{tabular}

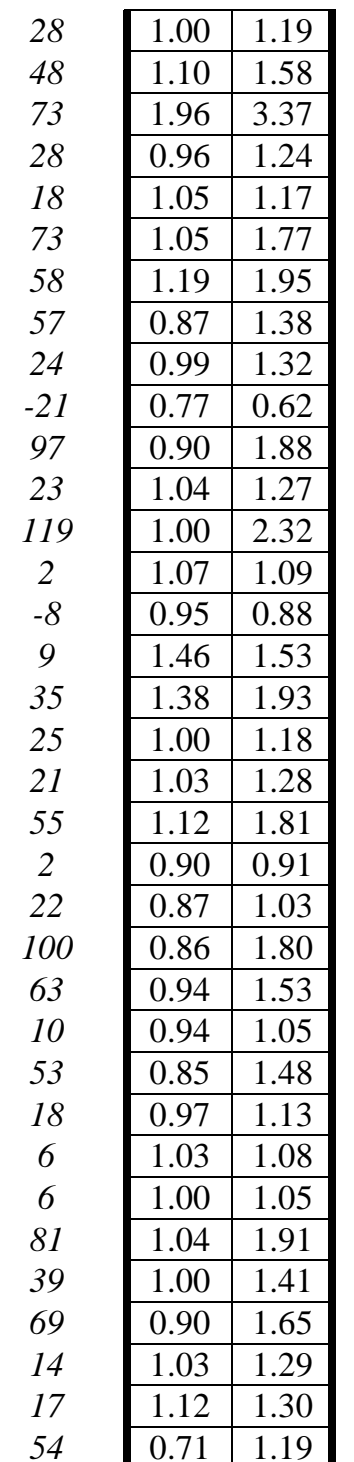




\begin{tabular}{|c|c|c|c|c|c|c|}
\hline 113 & $20 / 8 / 2012$ & $\mathrm{~N}$ & $7: 20$ & $16: 55$ & 0.26 & 0.37 \\
\hline 114 & $25 / 8 / 2012$ & $\mathrm{NE}$ & $9: 15$ & $11: 00$ & 1.16 & 1.52 \\
\hline 115 & $29 / 8 / 2012$ & $\mathrm{~N}$ & $8: 30$ & $13: 30$ & 0.89 & 0.90 \\
\hline 116 & $30 / 8 / 2012$ & $\mathrm{NE}$ & $8: 30$ & $13: 40$ & 0.87 & 1.25 \\
\hline 117 & $31 / 8 / 2012$ & $\mathrm{~N}$ & $10: 10$ & $11: 15$ & 1.09 & 1.82 \\
\hline 118 & $26 / 6 / 2013$ & $\mathrm{~N}$ & $9: 20$ & $11: 50$ & 1.05 & 1.49 \\
\hline 119 & $23 / 7 / 2013$ & $\mathrm{~N}$ & $8: 30$ & $14: 55$ & 0.79 & 0.66 \\
\hline 120 & $24 / 7 / 2013$ & $\mathrm{~N}$ & $8: 40$ & $11: 10$ & 1.09 & 1.28 \\
\hline 121 & $28 / 7 / 2013$ & $\mathrm{NE}$ & $9: 25$ & $12: 00$ & 1.02 & 1.25 \\
\hline 122 & $30 / 7 / 2013$ & $\mathrm{NE}$ & $9: 40$ & $11: 00$ & 1.08 & 1.33 \\
\hline 123 & $2 / 8 / 2013$ & $\mathrm{NE}$ & $9: 35$ & $10: 40$ & 0.92 & 1.10 \\
\hline 124 & $4 / 8 / 2013$ & $\mathrm{NE}$ & $8: 35$ & $10: 45$ & 1.11 & 1.67 \\
\hline 125 & $6 / 8 / 2013$ & $\mathrm{NE}$ & $8: 45$ & $10: 10$ & 1.01 & 1.60 \\
\hline 126 & $24 / 6 / 2014$ & $\mathrm{~N}$ & $9: 30$ & $13: 25$ & 1.10 & 1.21 \\
\hline Autumn & & & & & & \\
\hline 127 & $9 / 9 / 2008$ & $\mathrm{~N}$ & $10: 00$ & $15: 25$ & 1.05 & 1.19 \\
\hline 128 & $10 / 9 / 2008$ & $\mathrm{NE}$ & $10: 05$ & $14: 45$ & 0.92 & 0.86 \\
\hline 129 & $12 / 9 / 2008$ & $\mathrm{~N}$ & $9: 45$ & $14: 55$ & 1.30 & 1.75 \\
\hline 130 & $23 / 9 / 2008$ & $\mathrm{NW}$ & $9: 20$ & $12: 55$ & 1.01 & 1.31 \\
\hline 131 & $7 / 10 / 2008$ & $\mathrm{~N}$ & $10: 30$ & $13: 35$ & 1.28 & 1.50 \\
\hline 132 & $12 / 10 / 2008$ & $\mathrm{NE}$ & $10: 20$ & $13: 20$ & 0.95 & 0.96 \\
\hline 133 & $13 / 10 / 2008$ & $\mathrm{NE}$ & $12: 30$ & $16: 10$ & 1.04 & 1.05 \\
\hline 134 & $25 / 10 / 2008$ & $\mathrm{~N}$ & $13: 05$ & $17: 00$ & 1.00 & 1.28 \\
\hline 135 & $24 / 11 / 2008$ & $\mathrm{~W}$ & $12: 00$ & $14: 45$ & 1.05 & 0.96 \\
\hline 136 & $5 / 10 / 2009$ & $\mathrm{NW}$ & $7: 40$ & $17: 00$ & 1.02 & 2.43 \\
\hline 137 & $18 / 10 / 2009$ & $\mathrm{E}$ & $8: 05$ & $9: 35$ & 1.20 & 1.73 \\
\hline 138 & $19 / 10 / 2009$ & $\mathrm{~S} / \mathrm{SW}$ & $9: 20$ & $11: 45$ & 1.10 & 1.20 \\
\hline 139 & $20 / 10 / 2009$ & $\mathrm{~S} / \mathrm{SW}$ & $9: 35$ & $11: 35$ & 0.92 & 0.90 \\
\hline 140 & $24 / 9 / 2010$ & $\mathrm{~S} / \mathrm{SW}$ & $8: 30$ & $12: 35$ & 1.02 & 1.06 \\
\hline 141 & $6 / 10 / 2010$ & $\mathrm{~N}$ & $8: 20$ & $10: 00$ & 1.12 & 1.42 \\
\hline 142 & $9 / 10 / 2010$ & $\mathrm{~N}$ & $11: 25$ & $15: 45$ & 0.97 & 1.19 \\
\hline 143 & $12 / 10 / 2010$ & $\mathrm{~S} / \mathrm{SW}$ & $9: 55$ & $10: 25$ & 0.98 & 1.45 \\
\hline 144 & $15 / 10 / 2010$ & $\mathrm{~S} / \mathrm{SW}$ & $12: 25$ & $15: 50$ & 0.75 & 1.15 \\
\hline 145 & $28 / 10 / 2010$ & $\mathrm{~W}$ & $8: 50$ & $11: 50$ & 0.94 & 1.07 \\
\hline 146 & $1 / 11 / 2011$ & $\mathrm{~N}$ & $11: 20$ & $17: 45$ & 1.08 & 1.41 \\
\hline & & & & & & \\
\hline
\end{tabular}




\begin{tabular}{|c|c|c|c|c|c|c|}
\hline 147 & $10 / 11 / 2011$ & $\mathrm{~N}$ & $6: 35$ & $9: 40$ & 0.93 & 1.24 \\
\hline 148 & $13 / 11 / 2011$ & $\mathrm{NW}$ & $5: 35$ & $8: 45$ & 1.08 & 1.47 \\
\hline 149 & $9 / 9 / 2012$ & $\mathrm{~W}$ & $9: 20$ & $11: 35$ & 0.98 & 1.26 \\
\hline 150 & $20 / 9 / 2012$ & $\mathrm{~W}$ & $9: 00$ & $11: 20$ & 1.02 & 1.17 \\
\hline 151 & $22 / 9 / 2012$ & $\mathrm{NW}$ & $9: 35$ & $12: 45$ & 0.88 & 0.99 \\
\hline 152 & $3 / 11 / 2012$ & $\mathrm{~S} / \mathrm{SW}$ & $8: 25$ & $10: 10$ & 1.16 & 1.53 \\
\hline 153 & $10 / 11 / 2012$ & $\mathrm{~N}$ & $12: 50$ & $14: 55$ & 1.62 & 1.76 \\
\hline 154 & $21 / 11 / 2012$ & $\mathrm{~S} / \mathrm{SW}$ & $10: 30$ & $13: 30$ & 0.94 & 0.76 \\
\hline 155 & $18 / 10 / 2013$ & $\mathrm{NW}$ & $9: 00$ & $10: 20$ & 1.21 & 1.79 \\
\hline 156 & $20 / 10 / 2013$ & $\mathrm{~N}$ & $9: 25$ & $10: 00$ & 0.94 & 0.99 \\
\hline 157 & $8 / 11 / 2013$ & $\mathrm{NE}$ & $10: 00$ & $11: 05$ & 0.98 & 1.19 \\
\hline 158 & $12 / 9 / 2014$ & $\mathrm{~N}$ & $9: 15$ & $15: 55$ & 0.61 & 0.85 \\
\hline 159 & $19 / 10 / 2014$ & $\mathrm{~N}$ & $6: 35$ & $9: 35$ & 1.07 & 1.36 \\
\hline 160 & $20 / 10 / 2014$ & $\mathrm{~N}$ & $9: 10$ & $14: 30$ & 0.83 & 1.01 \\
\hline 161 & $1 / 11 / 2014$ & $\mathrm{NE}$ & $9: 10$ & $14: 00$ & 1.28 & 1.86 \\
\hline 162 & $14 / 11 / 2014$ & $\mathrm{NW}$ & $8: 40$ & $10: 20$ & 0.79 & 1.02 \\
\hline
\end{tabular}

\begin{tabular}{c|c|c|}
33 & 0.92 & 1.35 \\
36 & 1.13 & 1.67 \\
\hline 29 & 1.04 & 1.61 \\
15 & 1.13 & 1.30 \\
13 & 0.88 & 1.04 \\
\hline 32 & 1.21 & 2.32 \\
9 & 1.85 & 1.98 \\
\hline 19 & 1.33 & 1.92 \\
\hline 48 & 1.22 & 1.74 \\
5 & 0.99 & 1.17 \\
21 & 1.30 & 2.24 \\
39 & 0.53 & 0.85 \\
27 & 1.11 & 1.52 \\
22 & 0.99 & 1.41 \\
45 & 1.28 & 3.22 \\
29 & 0.82 & 1.14 \\
\hline
\end{tabular}

\begin{tabular}{|c|c|c|}
\hline 47 & 0.92 & 1.33 \\
48 & 1.12 & 1.61 \\
\hline 55 & 1.04 & 1.51 \\
15 & 1.12 & 1.25 \\
\hline 18 & 0.87 & 0.91 \\
\hline 92 & 1.18 & 1.93 \\
7 & 1.87 & 1.97 \\
\hline 4 & 1.41 & 2.25 \\
\hline 3 & 1.25 & 1.67 \\
18 & 0.99 & 1.07 \\
72 & 1.25 & 1.67 \\
60 & 0.55 & 0.85 \\
37 & 1.11 & 1.51 \\
\hline 42 & 1.04 & 1.53 \\
\hline 152 & 1.27 & 4.06 \\
39 & 0.79 & 1.05 \\
\hline
\end{tabular}

\begin{tabular}{c|c|c|}
45 & 0.92 & 1.31 \\
44 & 1.12 & 1.59 \\
45 & 1.02 & 1.41 \\
12 & 1.11 & 1.25 \\
\hline 5 & 0.87 & 0.86 \\
64 & 1.18 & 1.75 \\
5 & 1.88 & 1.94 \\
60 & 1.46 & 3.09 \\
34 & 1.26 & 1.74 \\
8 & 0.98 & 0.99 \\
34 & 1.18 & 1.31 \\
55 & 0.55 & 0.85 \\
36 & 1.12 & 1.48 \\
\hline 4 & 1.04 & 1.79 \\
220 & 1.30 & 4.76 \\
33 & 0.80 & 1.01 \\
\hline
\end{tabular}

\begin{tabular}{|c|c|c|}
\hline 42 & 0.97 & 1.31 \\
42 & 1.12 & 1.38 \\
38 & 1.02 & 1.39 \\
13 & 1.11 & 1.24 \\
\hline-1 & 0.87 & 0.83 \\
\hline 48 & 1.19 & 1.72 \\
\hline 3 & 1.90 & 1.92 \\
\hline 112 & 1.49 & 3.66 \\
\hline 38 & 1.25 & 1.78 \\
1 & 0.97 & 0.98 \\
11 & 1.16 & 1.24 \\
\hline 55 & 0.57 & 0.85 \\
32 & 1.12 & 1.47 \\
\hline 72 & 1.06 & 1.96 \\
\hline 266 & 1.30 & 5.03 \\
26 & 0.89 & 0.98 \\
\hline
\end{tabular}

\begin{tabular}{|c|c|c|}
35 & 1.00 & 1.29 \\
23 & 1.15 & 1.39 \\
36 & 0.99 & 1.36 \\
12 & 1.12 & 1.24 \\
-5 & 0.86 & 0.77 \\
\hline 45 & 1.26 & 1.68 \\
1 & 1.93 & 1.97 \\
146 & 1.61 & 6.00 \\
42 & 1.21 & 2.22 \\
1 & 0.97 & 0.93 \\
7 & 1.11 & 1.12 \\
49 & 0.61 & 0.85 \\
31 & 1.14 & 1.51 \\
85 & 1.10 & 2.61 \\
\hline 287 & 1.28 & 5.58 \\
10 & 0.88 & 0.97 \\
\hline
\end{tabular}

Table S3. Seasonal decoupling time $\left(t_{d e c}\right), t_{N d}$ when NPF starts to influence the cloud droplet number concentrations $\left(\mathrm{N}_{\mathrm{d}}\right)$, average $\mathrm{N}_{\mathrm{d}}$ and the estimated maximum supersaturation in the cloud ( $s_{\max }$ ) before (bef) being the time step between $t_{\text {dec }}$ and $t_{N d}$, and after (aft) being the time step between $t_{N d}$ and the end of $\mathrm{N}_{\mathrm{d}}$ production, and the increase of the $\mathrm{N}_{\mathrm{d}}$ and $s_{\max }(\%)$ observed after the $t_{N d}$. Two probability density functions (PDFs) of the characteristic updraft velocity are used with $\sigma_{\mathrm{w}}=0.3 \mathrm{~m} \mathrm{~s}^{-1}$ and $\sigma_{\mathrm{w}}=0.6 \mathrm{~m} \mathrm{~s}^{-1}$. Time is in LT.

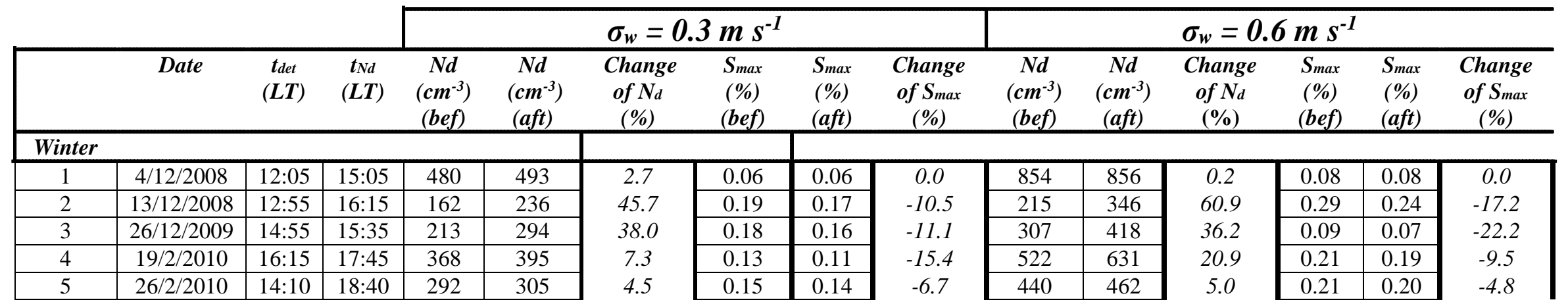




\begin{tabular}{|c|c|c|c|c|c|}
\hline 6 & $3 / 1 / 2011$ & $15: 55$ & $19: 50$ & 285 & 299 \\
\hline 7 & $6 / 1 / 2011$ & $15: 35$ & $17: 20$ & 190 & 227 \\
\hline 8 & $17 / 1 / 2011$ & $14: 20$ & $17: 50$ & 288 & 314 \\
\hline 9 & $19 / 1 / 2011$ & $17: 40$ & $18: 10$ & 236 & 338 \\
\hline 10 & $1 / 2 / 2011$ & $17: 00$ & $18: 15$ & 255 & 270 \\
\hline 11 & $7 / 2 / 2011$ & $14: 40$ & $15: 10$ & 473 & 483 \\
\hline 12 & $10 / 2 / 2011$ & $16: 30$ & $17: 25$ & 458 & 484 \\
\hline 13 & $11 / 2 / 2011$ & $17: 15$ & $18: 20$ & 331 & 452 \\
\hline 14 & $1 / 1 / 2012$ & - & - & - & - \\
\hline 15 & $26 / 1 / 2012$ & - & - & - & - \\
\hline 16 & $21 / 1 / 2013$ & $11: 50$ & $17: 50$ & 446 & 464 \\
\hline 17 & $23 / 1 / 2013$ & $12: 10$ & $14: 55$ & 476 & 497 \\
\hline 18 & $8 / 1 / 2014$ & $14: 30$ & $17: 35$ & 308 & 342 \\
\hline 19 & $10 / 1 / 2014$ & $12: 50$ & $15: 55$ & 436 & 487 \\
\hline 20 & $23 / 1 / 2014$ & $11: 25$ & $16: 05$ & 361 & 395 \\
\hline 21 & $30 / 1 / 2014$ & $12: 15$ & $15: 45$ & 451 & 484 \\
\hline 22 & $22 / 12 / 2014$ & $12: 30$ & $17: 50$ & 293 & 315 \\
\hline 23 & $11 / 1 / 2015$ & $11: 50$ & $16: 20$ & 300 & 312 \\
\hline 24 & $23 / 1 / 2015$ & $10: 50$ & $11: 45$ & 492 & 516 \\
\hline Spring & \multicolumn{1}{|c|}{} & & & \\
\hline 25 & $14 / 3 / 2009$ & $12: 30$ & $17: 20$ & 441 & 490 \\
\hline 26 & $19 / 3 / 2009$ & $14: 05$ & $17: 35$ & 462 & 491 \\
\hline 27 & $30 / 3 / 2009$ & $14: 40$ & $15: 20$ & 485 & 507 \\
\hline 28 & $22 / 5 / 2009$ & $10: 45$ & $15: 20$ & 469 & 493 \\
\hline 29 & $28 / 5 / 2009$ & $8: 35$ & $9: 25$ & 429 & 452 \\
\hline 30 & $13 / 3 / 2010$ & $11: 50$ & $12: 35$ & 311 & 315 \\
\hline 31 & $16 / 3 / 2010$ & $12: 55$ & $15: 15$ & 367 & 388 \\
\hline 32 & $18 / 3 / 2010$ & $14: 00$ & $15: 20$ & 348 & 380 \\
\hline 33 & $19 / 3 / 2010$ & $16: 05$ & $17: 45$ & 413 & 467 \\
\hline 34 & $22 / 3 / 2010$ & $12: 15$ & $16: 50$ & 457 & 463 \\
\hline 35 & $27 / 3 / 2010$ & $12: 55$ & $17: 25$ & 316 & 320 \\
\hline 36 & $30 / 3 / 2010$ & $11: 30$ & $12: 15$ & 246 & 297 \\
\hline 37 & $7 / 4 / 2010$ & $12: 15$ & $15: 55$ & 278 & 287 \\
\hline 38 & $8 / 4 / 2010$ & $13: 30$ & $14: 25$ & 355 & 439 \\
\hline 39 & $9 / 4 / 2010$ & $12: 05$ & $16: 20$ & 348 & 397 \\
\hline
\end{tabular}

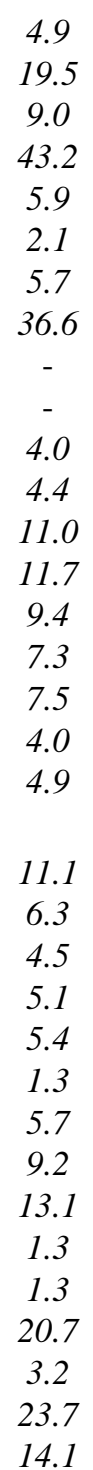

\begin{tabular}{|c|c|}
\hline 0.14 & 0.13 \\
\hline 0.20 & 0.19 \\
\hline 0.15 & 0.14 \\
\hline 0.18 & 0.15 \\
\hline 0.17 & 0.16 \\
\hline 0.13 & 0.11 \\
\hline 0.12 & 0.10 \\
\hline 0.14 & 0.13 \\
\hline- & - \\
\hline- & - \\
\hline 0.11 & 0.10 \\
\hline 0.12 & 0.11 \\
\hline 0.14 & 0.13 \\
\hline 0.13 & 0.11 \\
\hline 0.12 & 0.11 \\
\hline 0.15 & 0.14 \\
\hline 0.16 & 0.15 \\
\hline 0.12 & 0.11 \\
\hline 0.06 & 0.05 \\
\hline & \\
\hline 0.14 & 0.13 \\
\hline 0.15 & 0.14 \\
\hline 0.06 & 0.05 \\
\hline 0.12 & 0.10 \\
\hline 0.10 & 0.09 \\
\hline 0.13 & 0.13 \\
\hline 0.14 & 0.14 \\
\hline 0.14 & 0.14 \\
\hline 0.16 & 0.15 \\
\hline 0.11 & 0.12 \\
\hline 0.13 & 0.13 \\
\hline 0.14 & 0.12 \\
\hline 0.16 & 0.15 \\
\hline 0.13 & 0.10 \\
\hline 0.14 & 0.12 \\
\hline
\end{tabular}

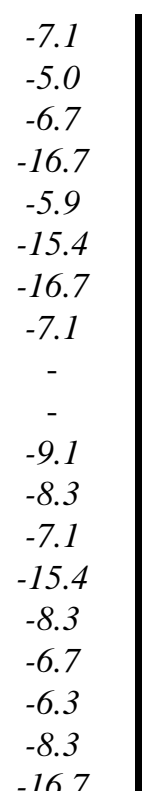

\begin{tabular}{|c|c|}
\hline 408 & 439 \\
\hline 275 & 344 \\
\hline 415 & 456 \\
\hline 336 & 507 \\
\hline 365 & 390 \\
\hline 698 & 813 \\
\hline 657 & 824 \\
\hline 507 & 617 \\
\hline- & - \\
\hline- & - \\
\hline 696 & 715 \\
\hline 823 & 849 \\
\hline 611 & 723 \\
\hline 573 & 689 \\
\hline 459 & 527 \\
\hline 572 & 651 \\
\hline 457 & 495 \\
\hline 464 & 477 \\
\hline 876 & 925 \\
\hline
\end{tabular}

7.6

25.1

9.9

50.9

6.8

16.5

25.4

21.7

-

2.7

3.2

18.3

20.2

14.8

13.8

8.3

2.8 5.6

\begin{tabular}{l|l|}
0.21 & 0.20 \\
\hline
\end{tabular}

\begin{tabular}{l|l|l|}
\hline 0.29 & 0.27 & -6.9 \\
\hline 0.21 & 0.19 \\
\hline
\end{tabular}

\begin{tabular}{ll|l}
0.21 & 0.19 & -9.5
\end{tabular}

\begin{tabular}{l|l|l|}
\hline 0.25 & 0.20 & -20.0 \\
\hline 0.25 & 0.24 \\
\hline
\end{tabular}

\begin{tabular}{l|l|l|}
\hline 0.25 & 0.20 & -20.0 \\
\hline 0.25 & 0.24 & -4
\end{tabular}

\begin{tabular}{l|l|l|}
\hline 0.16 & 0.15 & -6.3 \\
\hline 0.16 & 0.15 \\
\hline
\end{tabular}

\begin{tabular}{l|l|l|}
\hline 0.16 & 0.15 & -6.3 \\
\hline 0.20 & 0.18 \\
\hline
\end{tabular}

\begin{tabular}{l|l|l|}
\hline 0.20 & 0.18 & -10.0 \\
\hline
\end{tabular}

\begin{tabular}{|l|l|}
\hline & - \\
\hline- & - \\
\hline 0.15 & 0.13
\end{tabular}

\begin{tabular}{l|l|l|}
\hline 0.15 & 0.13 & -13.3 \\
\hline 0.17 & 0.16 & -5.9
\end{tabular}

\begin{tabular}{ll|l|}
\hline 0.17 & 0.16 & -5.9 \\
\hline 0.19 & 0.18 \\
\hline
\end{tabular}

\begin{tabular}{ll|l}
0.19 & 0.18 & -5.3
\end{tabular}

\begin{tabular}{l|l|l|}
\hline 0.22 & 0.20 & -9.1 \\
\hline 0.21 & 0.19 \\
\hline
\end{tabular}

\begin{tabular}{l|l|l|}
\hline 0.21 & 0.19 & -9.5 \\
\hline 0.19 & 0.18 \\
\hline
\end{tabular}

\begin{tabular}{l|l|l|}
\hline 0.19 & 0.18 & -5.3 \\
\hline 0.22 & 0.21
\end{tabular}

\begin{tabular}{ll|l|}
\hline 0.22 & 0.21 & -4.5 \\
\hline 0.19 & 0.18 \\
\hline
\end{tabular}

\begin{tabular}{l|l|l}
0.19 & 0.18 & -5.3
\end{tabular}

\begin{tabular}{l|l|l|}
0.19 & 0.18 & -5.3 \\
0.08 & 0.07 & -12.5
\end{tabular}

\begin{tabular}{|c|c|c|c|c|c|c|}
\hline-7.1 & 583 & 659 & 13.0 & 0.18 & 0.17 & -5.6 \\
\hline-6.7 & 547 & 631 & 15.4 & 0.20 & 0.19 & -5.0 \\
\hline-16.7 & 874 & 923 & 5.6 & 0.08 & 0.07 & -12.5 \\
\hline-16.7 & 614 & 679 & 10.6 & 0.16 & 0.15 & -6.3 \\
\hline-10.0 & 625 & 779 & 24.6 & 0.15 & 0.13 & -13.3 \\
\hline 0.0 & 473 & 481 & 1.7 & 0.19 & 0.18 & -5.3 \\
\hline 0.0 & 497 & 501 & 0.8 & 0.19 & 0.18 & -5.3 \\
\hline 0.0 & 459 & 471 & 2.6 & 0.20 & 0.19 & -5.0 \\
\hline-6.3 & 596 & 678 & 13.8 & 0.21 & 0.19 & -9.5 \\
\hline 9.1 & 620 & 763 & 23.1 & 0.15 & 0.16 & 6.7 \\
\hline 0.0 & 476 & 482 & 1.3 & 0.19 & 0.18 & -5.3 \\
\hline-14.3 & 365 & 443 & 21.4 & 0.19 & 0.17 & -10.5 \\
\hline-6.3 & 398 & 414 & 4.0 & 0.22 & 0.21 & -4.5 \\
\hline-23.1 & 497 & 652 & 31.2 & 0.17 & 0.15 & -11.8 \\
\hline-14.3 & 479 & 531 & 10.9 & 0.19 & 0.17 & -10.5 \\
\hline
\end{tabular}




\begin{tabular}{|c|c|c|c|c|c|}
\hline 40 & $15 / 4 / 2010$ & $14: 25$ & $16: 40$ & 310 & 325 \\
\hline 41 & $17 / 4 / 2010$ & $13: 35$ & $14: 40$ & 260 & 344 \\
\hline 42 & $29 / 4 / 2010$ & $13: 50$ & $15: 05$ & 379 & 463 \\
\hline 43 & $1 / 5 / 2010$ & $10: 40$ & $15: 15$ & 437 & 450 \\
\hline 44 & $30 / 5 / 2010$ & $9: 40$ & $14: 30$ & 337 & 368 \\
\hline 45 & 9/3/2011 & $11: 50$ & $14: 10$ & 267 & 341 \\
\hline 46 & $19 / 3 / 2011$ & $12: 05$ & $14: 30$ & 284 & 310 \\
\hline 47 & 29/3/2011 & $13: 00$ & $15: 15$ & 274 & 323 \\
\hline 48 & $12 / 4 / 2011$ & $13: 45$ & $19: 25$ & 336 & 483 \\
\hline 49 & $3 / 5 / 2011$ & $12: 30$ & $17: 05$ & 432 & 479 \\
\hline 50 & 4/5/2011 & $13: 50$ & $14: 45$ & 176 & 273 \\
\hline 51 & $14 / 5 / 2011$ & $10: 50$ & $13: 45$ & 336 & 361 \\
\hline 52 & $25 / 4 / 2012$ & - & - & - & - \\
\hline 53 & $1 / 5 / 2012$ & $12: 05$ & $16: 35$ & 418 & 441 \\
\hline 54 & $14 / 5 / 2012$ & $10: 55$ & $15: 25$ & 406 & 431 \\
\hline 55 & $6 / 3 / 2013$ & $12: 00$ & $17: 20$ & 234 & 243 \\
\hline 56 & $10 / 3 / 2013$ & $11: 45$ & $14: 25$ & 176 & 260 \\
\hline 57 & $13 / 3 / 2013$ & $13: 15$ & $16: 05$ & 214 & 252 \\
\hline 58 & $23 / 3 / 2013$ & $14: 50$ & $18: 20$ & 223 & 240 \\
\hline 59 & $24 / 3 / 2013$ & 9:30 & $18: 05$ & 300 & 379 \\
\hline 60 & $27 / 3 / 2013$ & $13: 50$ & $15: 45$ & 384 & 419 \\
\hline 61 & $5 / 4 / 2013$ & $12: 20$ & $15: 10$ & 236 & 254 \\
\hline 62 & $19 / 4 / 2013$ & $13: 10$ & $14: 00$ & 435 & 462 \\
\hline 63 & $21 / 4 / 2013$ & $15: 00$ & $17: 15$ & 469 & 492 \\
\hline 64 & $1 / 5 / 2013$ & $11: 45$ & 19:10 & 432 & 442 \\
\hline 65 & $22 / 5 / 2013$ & $15: 30$ & $17: 15$ & 228 & 241 \\
\hline 66 & $13 / 3 / 2014$ & 20:05 & 21:35 & 546 & 558 \\
\hline 67 & $21 / 3 / 2014$ & 21:05 & $21: 45$ & 453 & 464 \\
\hline 68 & $23 / 3 / 2014$ & $10: 15$ & $15: 50$ & 493 & 503 \\
\hline 69 & $30 / 3 / 2014$ & 13:05 & 18:15 & 322 & 327 \\
\hline 70 & $2 / 5 / 2014$ & $13: 35$ & $15: 20$ & 316 & 378 \\
\hline 71 & $21 / 5 / 2014$ & $10: 15$ & $16: 50$ & 440 & 456 \\
\hline 72 & 23/5/2014 & $12: 30$ & $15: 40$ & 418 & 427 \\
\hline 73 & $25 / 5 / 2014$ & $11: 25$ & $12: 50$ & 264 & 307 \\
\hline 74 & $26 / 5 / 2014$ & $11: 40$ & $16: 25$ & 350 & 379 \\
\hline
\end{tabular}

\begin{tabular}{|c|c|c|}
\hline 4.8 & 0.11 & 0.09 \\
\hline 32.3 & 0.12 & 0.09 \\
\hline 22.2 & 0.13 & 0.12 \\
\hline 3.0 & 0.11 & 0.10 \\
\hline 9.2 & 0.10 & 0.09 \\
\hline 27.7 & 0.14 & 0.13 \\
\hline 9.2 & 0.14 & 0.14 \\
\hline 17.9 & 0.12 & 0.12 \\
\hline 43.8 & 0.14 & 0.12 \\
\hline 10.9 & 0.15 & 0.13 \\
\hline 55.1 & 0.17 & 0.14 \\
\hline 7.4 & 0.13 & 0.12 \\
\hline- & - & - \\
\hline 5.5 & 0.11 & 0.10 \\
\hline 6.2 & 0.12 & 0.10 \\
\hline 3.8 & 0.18 & 0.17 \\
\hline 47.7 & 0.19 & 0.15 \\
\hline 17.8 & 0.17 & 0.16 \\
\hline 7.6 & 0.19 & 0.18 \\
\hline 26.3 & 0.14 & 0.13 \\
\hline 9.1 & 0.11 & 0.10 \\
\hline 7.6 & 0.13 & 0.12 \\
\hline 6.2 & 0.12 & 0.09 \\
\hline 4.9 & 0.09 & 0.08 \\
\hline 2.3 & 0.07 & 0.06 \\
\hline 5.7 & 0.11 & 0.10 \\
\hline 2.2 & 0.12 & 0.12 \\
\hline 2.4 & 0.13 & 0.13 \\
\hline 2.0 & 0.09 & 0.08 \\
\hline 1.6 & 0.16 & 0.15 \\
\hline 19.6 & 0.12 & 0.11 \\
\hline 3.6 & 0.09 & 0.08 \\
\hline 2.2 & 0.10 & 0.09 \\
\hline 16.3 & 0.14 & 0.12 \\
\hline 8.3 & 0.07 & 0.06 \\
\hline
\end{tabular}

\begin{tabular}{|c|c|c|}
\hline-18.2 & 380 & 433 \\
\hline-25.0 & 384 & 607 \\
\hline-7.7 & 561 & 663 \\
\hline-9.1 & 639 & 691 \\
\hline-10.0 & 451 & 548 \\
\hline-7.1 & 386 & 471 \\
\hline 0.0 & 466 & 509 \\
\hline 0.0 & 404 & 443 \\
\hline-14.3 & 503 & 652 \\
\hline-13.3 & 567 & 631 \\
\hline-17.6 & 268 & 409 \\
\hline-7.7 & 528 & 601 \\
\hline - & - & - \\
\hline-9.1 & 601 & 697 \\
\hline-16.7 & 582 & 656 \\
\hline-5.6 & 364 & 367 \\
\hline-21.1 & 269 & 435 \\
\hline-5.9 & 347 & 416 \\
\hline-5.3 & 366 & 394 \\
\hline-7.1 & 508 & 614 \\
\hline-9.1 & 632 & 698 \\
\hline-7.7 & 380 & 421 \\
\hline-25.0 & 600 & 758 \\
\hline-11.1 & 774 & 813 \\
\hline-14.3 & 751 & 756 \\
\hline-9.1 & 346 & 356 \\
\hline 0.0 & 983 & 1006 \\
\hline 0.0 & 588 & 622 \\
\hline-11.1 & 897 & 920 \\
\hline-6.3 & 486 & 517 \\
\hline-8.3 & 455 & 502 \\
\hline-11.1 & 732 & 787 \\
\hline-10.0 & 741 & 745 \\
\hline-14.3 & 356 & 434 \\
\hline-14.3 & 567 & 621 \\
\hline
\end{tabular}

\begin{tabular}{|c|c|c|}
\hline 13.9 & 0.16 & 0.14 \\
\hline 58.1 & 0.16 & 0.13 \\
\hline 18.2 & 0.17 & 0.16 \\
\hline 8.1 & 0.15 & 0.14 \\
\hline 21.5 & 0.14 & 0.13 \\
\hline 22.0 & 0.21 & 0.20 \\
\hline 9.2 & 0.19 & 0.19 \\
\hline 9.7 & 0.17 & 0.16 \\
\hline 29.6 & 0.19 & 0.16 \\
\hline 11.3 & 0.20 & 0.19 \\
\hline 52.6 & 0.23 & 0.19 \\
\hline 13.8 & 0.18 & 0.17 \\
\hline - & - & - \\
\hline 16.0 & 0.18 & 0.17 \\
\hline 12.7 & 0.19 & 0.18 \\
\hline 0.8 & 0.25 & 0.24 \\
\hline 61.7 & 0.26 & 0.19 \\
\hline 19.9 & 0.23 & 0.21 \\
\hline 7.7 & 0.25 & 0.24 \\
\hline 20.9 & 0.19 & 0.17 \\
\hline 10.4 & 0.17 & 0.16 \\
\hline 10.8 & 0.18 & 0.17 \\
\hline 26.3 & 0.18 & 0.15 \\
\hline 5.0 & 0.15 & 0.13 \\
\hline 0.7 & 0.10 & 0.09 \\
\hline 2.9 & 0.15 & 0.14 \\
\hline 2.3 & 0.16 & 0.16 \\
\hline 5.8 & 0.17 & 0.17 \\
\hline 2.6 & 0.13 & 0.12 \\
\hline 6.4 & 0.21 & 0.20 \\
\hline 10.3 & 0.15 & 0.15 \\
\hline 7.5 & 0.13 & 0.13 \\
\hline 0.5 & 0.14 & 0.13 \\
\hline 21.9 & 0.18 & 0.16 \\
\hline 9.5 & 0.12 & 0.11 \\
\hline
\end{tabular}




\begin{tabular}{|c|c|c|c|c|c|c|c|c|c|c|c|c|c|c|c|}
\hline 75 & $30 / 4 / 2015$ & $9: 35$ & $11: 05$ & 365 & 419 & 14.8 & 0.12 & 0.10 & -16.7 & 450 & 623 & 38.4 & 0.16 & 0.15 & -6.3 \\
\hline 76 & $2 / 5 / 2015$ & $10: 15$ & $12: 20$ & 384 & 417 & 8.6 & 0.12 & 0.10 & -16.7 & 504 & 656 & 30.2 & 0.16 & 0.15 & -6.3 \\
\hline \multicolumn{16}{|c|}{ Summer } \\
\hline 77 & $9 / 6 / 2008$ & $14: 45$ & $17: 00$ & 458 & 521 & 13.8 & 0.05 & 0.03 & -40.0 & 836 & 856 & 2.4 & 0.07 & 0.05 & -28.6 \\
\hline 78 & $15 / 6 / 2008$ & $9: 50$ & $14: 25$ & 378 & 387 & 2.4 & 0.07 & 0.07 & 0.0 & 594 & 602 & 1.3 & 0.12 & 0.11 & -8.3 \\
\hline 79 & $16 / 6 / 2008$ & $10: 20$ & $16: 20$ & 424 & 478 & 12.7 & 0.07 & 0.05 & -28.6 & 770 & 878 & 14.0 & 0.09 & 0.07 & -22.2 \\
\hline 80 & $20 / 6 / 2008$ & $12: 05$ & $16: 25$ & 434 & 476 & 9.7 & 0.06 & 0.05 & -16.7 & 794 & 881 & 11.0 & 0.08 & 0.06 & -25.0 \\
\hline 81 & $24 / 7 / 2008$ & $9: 30$ & $13: 40$ & 376 & 432 & 14.9 & 0.11 & 0.09 & -18.2 & 532 & 729 & 37.0 & 0.14 & 0.12 & -14.3 \\
\hline 82 & $25 / 7 / 2008$ & $11: 00$ & $12: 30$ & 422 & 432 & 2.4 & 0.10 & 0.10 & 0.0 & 645 & 754 & 16.9 & 0.14 & 0.13 & -7.1 \\
\hline 83 & $26 / 7 / 2008$ & $9: 50$ & $13: 40$ & 442 & 456 & 3.2 & 0.08 & 0.07 & -12.5 & 811 & 855 & 5.4 & 0.11 & 0.09 & -18.2 \\
\hline 84 & $30 / 7 / 2008$ & $12: 40$ & $16: 20$ & 446 & 457 & 2.5 & 0.07 & 0.06 & -14.3 & 781 & 794 & 1.7 & 0.10 & 0.09 & -10.0 \\
\hline 85 & $15 / 6 / 2009$ & $14: 10$ & $15: 40$ & 412 & 445 & 8.0 & 0.09 & 0.08 & -11.1 & 745 & 829 & 11.3 & 0.13 & 0.11 & -15.4 \\
\hline 86 & $19 / 6 / 2009$ & $13: 45$ & $15: 20$ & 279 & 329 & 17.9 & 0.12 & 0.12 & 0.0 & 453 & 498 & 9.9 & 0.16 & 0.15 & -6.3 \\
\hline 87 & $21 / 6 / 2009$ & $12: 45$ & $16: 20$ & 389 & 408 & 4.9 & 0.07 & 0.07 & 0.0 & 692 & 710 & 2.6 & 0.11 & 0.10 & -9.1 \\
\hline 88 & $22 / 7 / 2009$ & $14: 25$ & $17: 00$ & 370 & 403 & 8.9 & 0.10 & 0.09 & -10.0 & 510 & 647 & 26.9 & 0.14 & 0.13 & -7.1 \\
\hline 89 & $29 / 7 / 2009$ & $12: 05$ & $15: 55$ & 452 & 494 & 9.3 & 0.09 & 0.08 & -11.1 & 764 & 853 & 11.6 & 0.10 & 0.09 & -10.0 \\
\hline 90 & $7 / 6 / 2010$ & $12: 20$ & $13: 00$ & 441 & 453 & 2.7 & 0.08 & 0.07 & -12.5 & 761 & 805 & 5.8 & 0.13 & 0.12 & -7.7 \\
\hline 91 & $5 / 7 / 2010$ & $15: 15$ & $19: 25$ & 424 & 431 & 1.7 & 0.06 & 0.06 & 0.0 & 718 & 728 & 1.4 & 0.09 & 0.09 & 0.0 \\
\hline 92 & $24 / 7 / 2010$ & $10: 35$ & $14: 00$ & 399 & 412 & 3.3 & 0.06 & 0.05 & -16.7 & 712 & 732 & 2.8 & 0.08 & 0.07 & -12.5 \\
\hline 93 & $18 / 8 / 2010$ & 9:55 & $10: 45$ & 361 & 380 & 5.3 & 0.07 & 0.06 & -14.3 & 639 & 680 & 6.4 & 0.11 & 0.09 & -18.2 \\
\hline 94 & $30 / 8 / 2010$ & $12: 30$ & $14: 30$ & 310 & 333 & 7.4 & 0.11 & 0.10 & -9.1 & 664 & 702 & 5.7 & 0.13 & 0.12 & -7.7 \\
\hline 95 & $27 / 6 / 2011$ & $12: 40$ & $14: 15$ & 419 & 441 & 5.3 & 0.10 & 0.09 & -10.0 & 671 & 722 & 7.6 & 0.14 & 0.13 & -7.1 \\
\hline 96 & $11 / 7 / 2011$ & $10: 55$ & $12: 05$ & 406 & 421 & 3.7 & 0.09 & 0.08 & -11.1 & 622 & 703 & 13.0 & 0.14 & 0.13 & -7.1 \\
\hline 97 & $16 / 7 / 2011$ & $10: 55$ & $12: 30$ & 382 & 397 & 3.9 & 0.10 & 0.10 & 0.0 & 502 & 569 & 13.3 & 0.12 & 0.11 & -8.3 \\
\hline 98 & $18 / 7 / 2011$ & $9: 55$ & $12: 15$ & 393 & 417 & 6.1 & 0.08 & 0.07 & -12.5 & 701 & 757 & 8.0 & 0.10 & 0.09 & -10.0 \\
\hline 99 & $22 / 7 / 2011$ & 9:05 & $15: 10$ & 421 & 441 & 4.8 & 0.07 & 0.06 & -14.3 & 723 & 771 & 6.6 & 0.10 & 0.09 & -10.0 \\
\hline 100 & $26 / 7 / 2011$ & $13: 20$ & $14: 40$ & 438 & 451 & 3.0 & 0.09 & 0.08 & -11.1 & 807 & 831 & 3.0 & 0.11 & 0.10 & -9.1 \\
\hline 101 & $14 / 6 / 2012$ & $11: 25$ & $13: 20$ & 431 & 449 & 4.2 & 0.06 & 0.05 & -16.7 & 808 & 849 & 5.1 & 0.08 & 0.07 & -12.5 \\
\hline 102 & $16 / 6 / 2012$ & $9: 40$ & $15: 05$ & 423 & 438 & 3.5 & 0.07 & 0.06 & -14.3 & 751 & 793 & 5.6 & 0.10 & 0.09 & -10.0 \\
\hline 103 & $17 / 6 / 2012$ & $15: 20$ & $17: 00$ & 385 & 397 & 3.1 & 0.09 & 0.09 & 0.0 & 656 & 671 & 2.3 & 0.14 & 0.13 & -7.1 \\
\hline 104 & $18 / 6 / 2012$ & $10: 25$ & $11: 25$ & 278 & 353 & 27.0 & 0.12 & 0.11 & -8.3 & 435 & 473 & 8.7 & 0.16 & 0.15 & -6.3 \\
\hline 105 & $19 / 6 / 2012$ & $12: 40$ & $14: 45$ & 410 & 423 & 3.2 & 0.09 & 0.08 & -11.1 & 754 & 782 & 3.7 & 0.12 & 0.12 & 0.0 \\
\hline 106 & $24 / 6 / 2012$ & $11: 15$ & $16: 25$ & 444 & 453 & 2.0 & 0.05 & 0.05 & 0.0 & 795 & 808 & 1.6 & 0.07 & 0.07 & 0.0 \\
\hline 107 & $27 / 6 / 2012$ & $9: 50$ & $12: 25$ & 413 & 438 & 6.1 & 0.06 & 0.05 & -16.7 & 733 & 782 & 6.7 & 0.08 & 0.07 & -12.5 \\
\hline 108 & 29/6/2012 & $10: 30$ & $16: 50$ & 411 & 428 & 4.1 & 0.08 & 0.07 & -12.5 & 698 & 764 & 9.5 & 0.12 & 0.11 & -8.3 \\
\hline
\end{tabular}




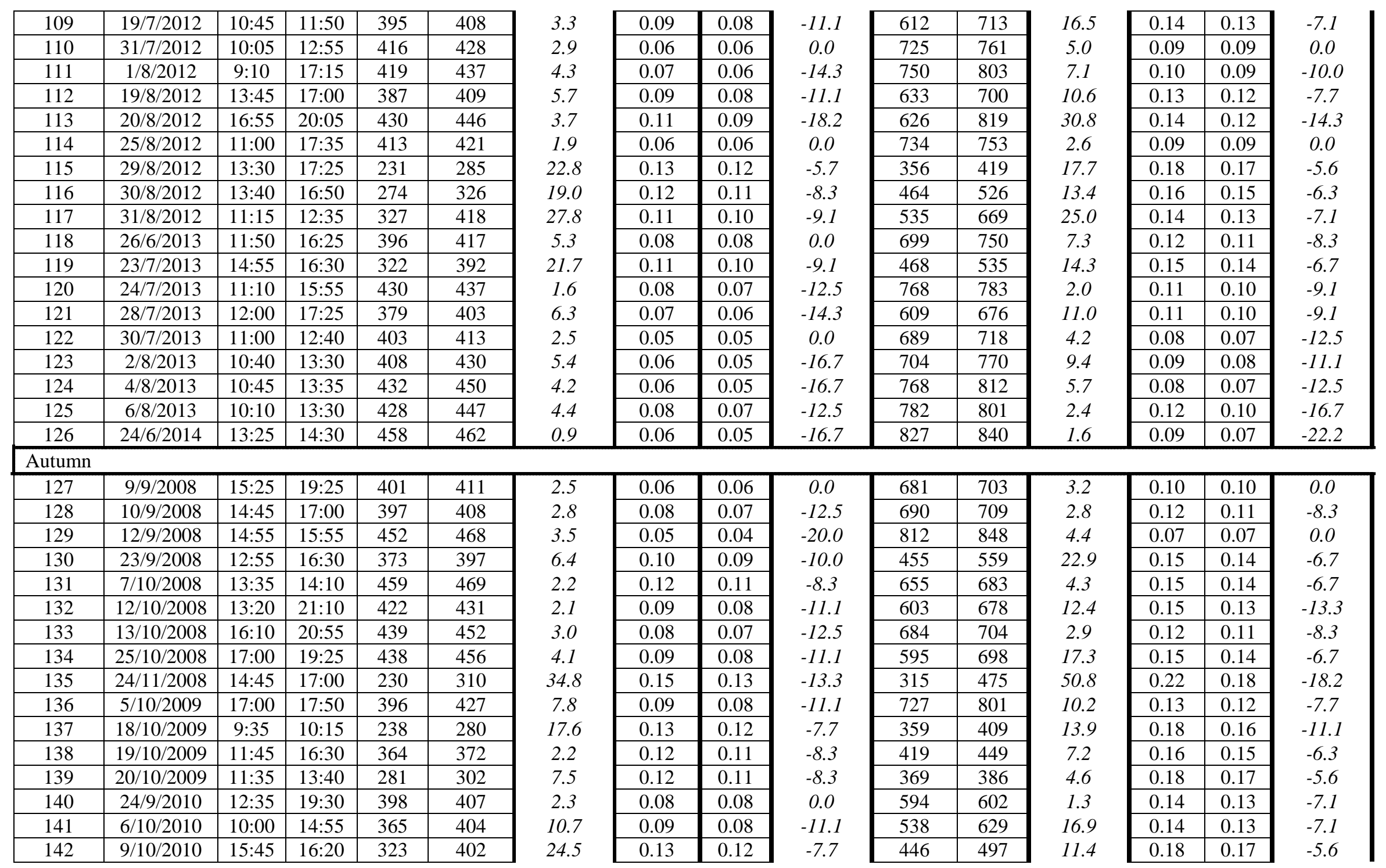




\begin{tabular}{|c|c|c|c|c|c|}
\hline 143 & $12 / 10 / 2010$ & $10: 25$ & $11: 35$ & 200 & 222 \\
\hline 144 & $15 / 10 / 2010$ & $15: 50$ & $19: 40$ & 203 & 229 \\
\hline 145 & $28 / 10 / 2010$ & $11: 50$ & $16: 15$ & 157 & 183 \\
\hline 146 & $1 / 11 / 2011$ & $17: 45$ & $18: 55$ & 352 & 439 \\
\hline 147 & $10 / 11 / 2011$ & $9: 40$ & $10: 55$ & 259 & 311 \\
\hline 148 & $13 / 11 / 2011$ & $8: 45$ & $10: 25$ & 409 & 463 \\
\hline 149 & $9 / 9 / 2012$ & $11: 35$ & $12: 50$ & 406 & 422 \\
\hline 150 & $20 / 9 / 2012$ & $11: 20$ & $16: 50$ & 428 & 446 \\
\hline 151 & $22 / 9 / 2012$ & $12: 45$ & $16: 50$ & 393 & 416 \\
\hline 152 & $3 / 11 / 2012$ & $10: 10$ & $13: 40$ & 413 & 421 \\
\hline 153 & $10 / 11 / 2012$ & $14: 55$ & $16: 35$ & 240 & 309 \\
\hline 154 & $21 / 11 / 2012$ & $13: 30$ & $17: 25$ & 223 & 279 \\
\hline 155 & $18 / 10 / 2013$ & $10: 20$ & $13: 20$ & 266 & 365 \\
\hline 156 & $20 / 10 / 2013$ & $10: 00$ & $12: 15$ & 413 & 459 \\
\hline 157 & $8 / 11 / 2013$ & $11: 05$ & $13: 15$ & 209 & 244 \\
\hline 158 & $12 / 9 / 2014$ & $15: 55$ & $18: 10$ & 391 & 407 \\
\hline 159 & $19 / 10 / 2014$ & $9: 35$ & $10: 55$ & 259 & 301 \\
\hline 160 & $20 / 10 / 2014$ & $14: 30$ & $16: 40$ & 368 & 421 \\
\hline 161 & $1 / 11 / 2014$ & $14: 00$ & $17: 25$ & 425 & 455 \\
\hline 162 & $14 / 11 / 2014$ & $10: 20$ & $13: 10$ & 296 & 402 \\
\hline
\end{tabular}

\begin{tabular}{l|l|l|} 
11.0 & 0.12 & 0.11 \\
\cline { 2 - 3 } 12.8 & 0.14 & 0.13 \\
\cline { 2 - 3 } 16.6 & 0.19 & 0.18 \\
\hline 24.7 & 0.14 & 0.13 \\
\cline { 2 - 3 } 20.1 & 0.13 & 0.12 \\
\cline { 2 - 3 } 13.2 & 0.10 & 0.09 \\
\hline 3.9 & 0.10 & 0.09 \\
\cline { 2 - 3 } 4.2 & 0.07 & 0.06 \\
\cline { 2 - 3 } 5.9 & 0.10 & 0.09 \\
1.9 & 0.11 & 0.10 \\
\hline 28.8 & 0.15 & 0.14 \\
\cline { 2 - 3 } 25.1 & 0.14 & 0.13 \\
37.2 & 0.13 & 0.12 \\
\hline 11.1 & 0.12 & 0.11 \\
\cline { 2 - 3 } 16.7 & 0.17 & 0.16 \\
\hline 4.1 & 0.10 & 0.06 \\
\hline 16.2 & 0.14 & 0.13 \\
\cline { 2 - 3 } 14.4 & 0.09 & 0.08 \\
\hline 7.1 & 0.08 & 0.07 \\
\cline { 2 - 3 } 35.8 & 0.14 & 0.13 \\
\hline
\end{tabular}

\begin{tabular}{|l|l|l|}
\hline-8.3 & 294 & 330 \\
\cline { 2 - 3 }-7.1 & 291 & 318 \\
\cline { 2 - 3 }-5.3 & 202 & 247 \\
\hline-7.1 & 527 & 631 \\
\hline-7.7 & 376 & 431 \\
\hline-10.0 & 606 & 739 \\
-10.0 & 569 & 693 \\
\hline-14.3 & 705 & 728 \\
\hline-10.0 & 479 & 568 \\
-9.1 & 579 & 694 \\
\hline-6.7 & 385 & 515 \\
\hline-7.1 & 288 & 439 \\
-7.7 & 383 & 443 \\
\hline-8.3 & 576 & 619 \\
\hline-5.9 & 365 & 456 \\
\hline-40.0 & 573 & 602 \\
\hline-7.1 & 378 & 427 \\
\hline-11.1 & 671 & 718 \\
\hline-12.5 & 626 & 735 \\
\hline-7.1 & 483 & 569 \\
\hline
\end{tabular}

12.2
9.3
22.3
19.7
14.6
21.9
21.8
3.3
18.6
19.9
33.8
52.4
15.7
7.5
24.9
5.1
13.0
7.0
17.4
17.8

\begin{tabular}{|l|l|}
\hline 0.17 & 0.16 \\
\hline 0.20 & 0.19 \\
\hline 0.28 & 0.26 \\
\hline 0.20 & 0.18 \\
\hline 0.18 & 0.17 \\
\hline 0.15 & 0.14 \\
\hline 0.14 & 0.13 \\
\hline 0.11 & 0.10 \\
\hline 0.14 & 0.13 \\
\hline 0.14 & 0.13 \\
\hline 0.21 & 0.19 \\
\hline 0.22 & 0.18 \\
\hline 0.18 & 0.16 \\
\hline 0.16 & 0.15 \\
\hline 0.23 & 0.20 \\
\hline 0.10 & 0.09 \\
\hline 0.18 & 0.17 \\
\hline 0.13 & 0.12 \\
\hline 0.15 & 0.12 \\
\hline 0.19 & 0.17 \\
\hline
\end{tabular}

$-5.9$

Table S4. Variance of $\mathrm{N}_{\mathrm{d}}$ and relative contribution to this variance of aerosol number ( $\mathrm{N}_{\text {total }}$ ) and chemical composition ( $\kappa$ ) during all the NPF events from May 2012 to May 2015, based on the ACSM measurements.

\begin{tabular}{|c|c|c|c|c|c|c|c|}
\hline \multirow{2}{*}{\multicolumn{2}{|c|}{ Date }} & \multicolumn{2}{|c|}{ Variance $\mathbf{N}_{\mathrm{d}}$} & \multicolumn{2}{|c|}{ Contribution of $\boldsymbol{\kappa}$} & \multicolumn{2}{|c|}{ Contribution of $\mathrm{N}_{\text {total }}$} \\
\hline & & $\sigma_{w}=0.3 \mathrm{~m} \mathrm{~s}^{-1}$ & $\sigma_{w}=0.6 \mathrm{~m} \mathrm{~s}^{-1}$ & $\sigma_{w}=0.3 \mathrm{~m} \mathrm{~s}^{-1}$ & $\sigma_{w}=0.6 \mathrm{~m} \mathrm{~s}^{-1}$ & $\sigma_{w}=0.3 \mathrm{~m} \mathrm{~s}^{-1}$ & $\sigma_{w}=0.6 \mathrm{~m} \mathrm{~s}^{-1}$ \\
\hline \multicolumn{8}{|c|}{ Winter } \\
\hline 1 & $22 / 12 / 2014$ & 94.4 & 146.9 & $12.0 \%$ & $15.0 \%$ & $88.0 \%$ & $85.0 \%$ \\
\hline 2 & 11/1/2015 & 94.7 & 182.8 & $14.0 \%$ & $6.0 \%$ & $86.0 \%$ & $94.0 \%$ \\
\hline 3 & $23 / 1 / 2015$ & 205.2 & 567.9 & $1.0 \%$ & $1.0 \%$ & $99.0 \%$ & $99.0 \%$ \\
\hline \multicolumn{2}{|c|}{ Spring } & & & & & & \\
\hline
\end{tabular}




\begin{tabular}{|c|c|c|c|c|c|c|c|}
\hline 4 & $6 / 3 / 2013$ & 48.9 & 81.4 & $6.0 \%$ & $5.0 \%$ & $94.0 \%$ & $95.0 \%$ \\
\hline 5 & $10 / 3 / 2013$ & 70.6 & 117.1 & $24.0 \%$ & $22.0 \%$ & $76.0 \%$ & $78.0 \%$ \\
\hline 6 & $13 / 3 / 2013$ & 59.4 & 92.7 & $28.0 \%$ & $27.0 \%$ & $72.0 \%$ & $73.0 \%$ \\
\hline 7 & 23/3/2013 & 77.7 & 133.6 & $15.0 \%$ & $14.0 \%$ & $85.0 \%$ & $86.0 \%$ \\
\hline 8 & $24 / 3 / 2013$ & 177.8 & 243.6 & $12.0 \%$ & $7.0 \%$ & $88.0 \%$ & $93.0 \%$ \\
\hline 9 & $27 / 3 / 2013$ & 191.1 & 262.6 & $21.0 \%$ & $14.0 \%$ & $79.0 \%$ & $86.0 \%$ \\
\hline 10 & $5 / 4 / 2013$ & 185.7 & 311.2 & $6.0 \%$ & $5.0 \%$ & $94.0 \%$ & $95.0 \%$ \\
\hline 11 & $19 / 4 / 2013$ & 155.8 & 288.4 & $0.0 \%$ & $1.0 \%$ & $100.0 \%$ & $99.0 \%$ \\
\hline 12 & $21 / 4 / 2013$ & 59.7 & 300.3 & $0.0 \%$ & $4.0 \%$ & $100.0 \%$ & $96.0 \%$ \\
\hline 13 & $1 / 5 / 2013$ & 16.7 & 41.8 & $1.0 \%$ & $0.0 \%$ & $99.0 \%$ & $100.0 \%$ \\
\hline 14 & $22 / 5 / 2013$ & 56.2 & 150.4 & $20.0 \%$ & $7.0 \%$ & $80.0 \%$ & $93.0 \%$ \\
\hline 15 & $30 / 4 / 2015$ & 208.2 & 221.7 & $3.0 \%$ & $5.0 \%$ & $97.0 \%$ & $95.0 \%$ \\
\hline 16 & $2 / 5 / 2015$ & 83.4 & 151.2 & $5.0 \%$ & $8.0 \%$ & $95.0 \%$ & $92.0 \%$ \\
\hline \multicolumn{8}{|c|}{ Summer } \\
\hline 17 & 14/6/2012 & 11.8 & 40.1 & $4.0 \%$ & $6.0 \%$ & $96.0 \%$ & $94.0 \%$ \\
\hline 18 & 16/6/2012 & 8.3 & 21.2 & $7.0 \%$ & $5.0 \%$ & $93.0 \%$ & $95.0 \%$ \\
\hline 19 & 17/6/2012 & 15.1 & 79.6 & $11.0 \%$ & $23.0 \%$ & $89.0 \%$ & $77.0 \%$ \\
\hline 20 & $24 / 6 / 2012$ & 14.9 & 46.3 & $1.0 \%$ & $0.0 \%$ & $99.0 \%$ & $100.0 \%$ \\
\hline 21 & 27/6/2012 & 28.8 & 73.3 & $1.0 \%$ & $1.0 \%$ & $99.0 \%$ & $99.0 \%$ \\
\hline 22 & 29/6/2012 & 20.2 & 78.5 & $2.0 \%$ & $6.0 \%$ & $98.0 \%$ & $94.0 \%$ \\
\hline 23 & 19/7/2012 & 12.1 & 96.1 & $5.0 \%$ & $21.0 \%$ & $95.0 \%$ & $79.0 \%$ \\
\hline 24 & $31 / 7 / 2012$ & 28.3 & 90.2 & $1.0 \%$ & $1.0 \%$ & $99.0 \%$ & $99.0 \%$ \\
\hline 25 & 1/8/2012 & 13.5 & 35.8 & $7.0 \%$ & $3.0 \%$ & $93.0 \%$ & $97.0 \%$ \\
\hline 26 & 19/8/2012 & 28.9 & 109.6 & $11.0 \%$ & $18.0 \%$ & $89.0 \%$ & $82.0 \%$ \\
\hline 27 & 20/8/2012 & 93.5 & 96.1 & $53.0 \%$ & $37.0 \%$ & $47.0 \%$ & $63.0 \%$ \\
\hline 28 & $25 / 8 / 2012$ & 26.5 & 74.8 & $2.0 \%$ & $6.0 \%$ & $98.0 \%$ & $94.0 \%$ \\
\hline 29 & 29/8/2012 & 172.6 & 147.0 & $10.0 \%$ & $10.0 \%$ & $90.0 \%$ & $90.0 \%$ \\
\hline 30 & $30 / 8 / 2012$ & 176.7 & 150.4 & $12.0 \%$ & $12.0 \%$ & $88.0 \%$ & $88.0 \%$ \\
\hline 31 & $31 / 8 / 2012$ & 36.1 & 120.1 & $31.0 \%$ & $42.0 \%$ & $69.0 \%$ & $58.0 \%$ \\
\hline 32 & $26 / 6 / 2013$ & 11.3 & 59.2 & $8.0 \%$ & $45.0 \%$ & $92.0 \%$ & $55.0 \%$ \\
\hline
\end{tabular}




\begin{tabular}{|c|c|c|c|c|c|c|c|}
\hline 33 & $23 / 7 / 2013$ & 47.3 & 513.3 & $23.0 \%$ & $34.0 \%$ & $77.0 \%$ & $66.0 \%$ \\
\hline 34 & $24 / 7 / 2013$ & 20.2 & 290.2 & $2.0 \%$ & $2.0 \%$ & $98.0 \%$ & $98.0 \%$ \\
\hline 35 & 28/7/2013 & 32.9 & 85.3 & $2.0 \%$ & $1.0 \%$ & $98.0 \%$ & $99.0 \%$ \\
\hline 36 & $30 / 7 / 2013$ & 13.7 & 32.5 & $3.0 \%$ & $4.0 \%$ & $97.0 \%$ & $96.0 \%$ \\
\hline 37 & $2 / 8 / 2013$ & 9.1 & 20.8 & $3.0 \%$ & $3.0 \%$ & $97.0 \%$ & $97.0 \%$ \\
\hline 38 & $4 / 8 / 2013$ & 10.6 & 26.9 & $2.0 \%$ & $3.0 \%$ & $98.0 \%$ & $97.0 \%$ \\
\hline 39 & $6 / 8 / 2013$ & 4.7 & 38.2 & $9.0 \%$ & $38.0 \%$ & $91.0 \%$ & $62.0 \%$ \\
\hline \multicolumn{8}{|c|}{ Autumn } \\
\hline 40 & 20/9/2012 & 44.1 & 119.2 & $1.0 \%$ & $1.0 \%$ & $99.0 \%$ & $99.0 \%$ \\
\hline 41 & 22/9/2012 & 22.5 & 71.5 & $1.0 \%$ & $13.0 \%$ & $99.0 \%$ & $87.0 \%$ \\
\hline 42 & 3/11/2012 & 115.3 & 224.1 & $2.0 \%$ & $6.0 \%$ & $98.0 \%$ & $94.0 \%$ \\
\hline 43 & 18/10/2013 & 96.6 & 96.8 & $7.0 \%$ & $7.0 \%$ & $93.0 \%$ & $93.0 \%$ \\
\hline 44 & 20/10/2013 & 40.2 & 54.4 & $17.0 \%$ & $15.0 \%$ & $83.0 \%$ & $85.0 \%$ \\
\hline 45 & 8/11/2013 & 43.4 & 77.9 & $17.0 \%$ & $21.0 \%$ & $83.0 \%$ & $79.0 \%$ \\
\hline 46 & $12 / 9 / 2014$ & 38.5 & 108.6 & $1.0 \%$ & $0.0 \%$ & $99.0 \%$ & $100.0 \%$ \\
\hline 47 & 1/11/2014 & 251.6 & 837.1 & $1.0 \%$ & $1.0 \%$ & $99.0 \%$ & $99.0 \%$ \\
\hline 48 & $14 / 11 / 2014$ & 192.9 & 274.2 & $6.0 \%$ & $5.0 \%$ & $94.0 \%$ & $95.0 \%$ \\
\hline
\end{tabular}

Table S5. Seasonal percentage change of the estimated $s_{\max }$ (maximum supersaturation in the cloud) and $\mathrm{N}_{\mathrm{d}}$ (cloud droplet number concentration) before and after the $t_{N d}$, the variance of $\mathrm{N}_{\mathrm{d}}$, the relative contribution of the total aerosol number $\left(\mathrm{N}_{\text {total }}\right)$ and the hygroscopicity ( $\kappa$ ) to the droplet number after the $t_{N d}$ calculated according to the approach described in the main text. Two probability density functions (PDFs) of the characteristic updraft velocity are used with $\sigma_{\mathrm{w}}=0.3 \mathrm{~m} \mathrm{~s}^{-1}$ and $\sigma_{\mathrm{w}}=0.6 \mathrm{~m} \mathrm{~s}^{-1}$.

\begin{tabular}{ll|c|c|c|c}
\hline & Winter & Spring & Summer & Autumn \\
\hline \multicolumn{7}{c|}{$\boldsymbol{\sigma}_{\mathbf{w}}=\mathbf{0 . 3} \mathbf{~ m ~ s}^{-\mathbf{1}}$} \\
\hline $\begin{array}{l}\text { Percentage } \\
\text { the } s_{\max }(\%)\end{array}$ & change of & $(-) 9.8$ & $(-) 9.7$ & $(-) 10.0$ & (-) 10.0
\end{tabular}




\begin{tabular}{|c|c|c|c|c|}
\hline $\begin{array}{l}\text { Percentage change of } \\
\text { the } N_{d}\left(\mathrm{~cm}^{-3}\right)\end{array}$ & 12.6 & 12.0 & 7.0 & 12.5 \\
\hline Variance of $\mathrm{N}_{\mathrm{d}}$ & 131 & 111 & 32 & 91 \\
\hline Contribution of $\kappa$ & 9.0 & 11.0 & 9.0 & 6.0 \\
\hline \multirow[t]{2}{*}{ Contribution of $\mathrm{N}_{\text {total }}$} & 91.0 & 89.0 & 91.0 & 94.0 \\
\hline & \multicolumn{4}{|c|}{$\sigma_{\mathrm{w}}=0.6 \mathrm{~m} \mathrm{~s}^{-1}$} \\
\hline $\begin{array}{l}\text { Percentage change of } \\
\text { the } s_{\max }(\%)\end{array}$ & $(-) 8.9$ & $(-) 7.5$ & (-) 10.0 & $(-) 6.3$ \\
\hline $\begin{array}{l}\text { Percentage change of } \\
\text { the } N_{d}\left(\mathrm{~cm}^{-3}\right)\end{array}$ & 16.7 & 15.3 & 9.0 & 15.0 \\
\hline Variance of $\mathrm{N}_{\mathrm{d}}$ & 299 & 175 & 101 & 205 \\
\hline Contribution of $\kappa$ & 7.0 & 9.0 & 14.0 & 8.0 \\
\hline Contribution of $\mathrm{N}_{\text {total }}$ & 93.0 & 91.0 & 86.0 & 92.0 \\
\hline
\end{tabular}

